\title{
High-enthalpy hypersonic flows
}

\author{
Joseph J. S. Shang ${ }^{1 *}$ (D) and Hong Yan ${ }^{2}$
}

\author{
* Correspondence: joseph.shang@ \\ wright.edu; josephjshang@gmail. \\ com \\ ${ }^{1}$ Wright State University, 3640 \\ Colonel Glenn Highway, Dayton, \\ $\mathrm{OH}$ 45435, USA \\ Full list of author information is \\ available at the end of the article
}

\begin{abstract}
Nearly all illuminating classic hypersonic flow theories address aerodynamic phenomena as a perfect gas in the high-speed range and at the upper limit of continuum gas domain. The hypersonic flow is quantitatively defined by the Mach number independent principle, which is derived from the asymptotes of the Rankine-Hugoniot relationship. However, most hypersonic flows encounter strong shock-wave compressions resulting in a high enthalpy gas environment that always associates with nonequilibrium thermodynamic and quantum chemical-physics phenomena. Under this circumstance, the theoretic linkage between the microscopic particle dynamics and macroscopic thermodynamics properties of gas is lost. When the air mixture is ionized to become an electrically conducting medium, the governing physics now ventures into the regimes of quantum physics and electromagnetics. Therefore, the hypersonic flows are no longer a pure aerodynamics subject but a multidisciplinary science. In order to better understand the realistic hypersonic flows, all pertaining disciplines such as the nonequilibrium chemical kinetics, quantum physics, radiative heat transfer, and electromagnetics need to bring forth.

Keywords: Hypersonic flow, Nonequilibrium chemical kinetics, Ionization, Quantum mechanics, Electromagnetics, Radiation
\end{abstract}

\section{Introduction}

These invaluable classic theories such as the rarefied gas dynamics, Newtonian flow theory, hypersonic similitude, law of cross-section, blast wave analogue, as well as, the Mach wave and vorticity interactions at the leading edges are developed from remarkable insights and firmly established by rigorous analyses. The collective accomplishments contribute to the fundamental knowledge of aerodynamics and have opened avenues in search of better understanding and innovation. Meanwhile, these theories have withheld the test of time and set excellent standards for basic research in science.

Most hypersonic flows are observed in the upper atmosphere, in the stratospheric and the lower altitude of the mesospheric layer. In these environments, the mean-freepath between molecule collisions approaches that of the characteristic length of the investigated configuration. In terms of the Knudson number, its value is approaching unity [1]. From the gas kinetic theory, the mean-free-path $\lambda=1 / \sqrt{2} n \pi \sigma^{2}$ is inversely proportional to the particle number density $n$, and collision cross section $\sigma$. Its dimension can be determined from the averaged molecule speed, $u_{m}=\sqrt{8 \mathrm{kT} / \pi m}$ and mo-

(c) The Author(s). 2020, corrected publication 2020. Open Access This article is licensed under a Creative Commons Attribution 4.0 International License, which permits use, sharing, adaptation, distribution and reproduction in any medium or format, as long as you give appropriate credit to the original author(s) and the source, provide a link to the Creative Commons licence, and indicate if changes were made. The images or other third party material in this article are included in the article's Creative Commons licence, unless indicated otherwise in a credit line to the material. If material is not included in the article's Creative Commons licence and your intended use is not permitted by statutory regulation or exceeds the permitted use, you will need to obtain permission directly from the copyright holder. To view a copy of this licence, visit http://creativecommons.org/licenses/by/4.0/. 
lecular collision frequency $n_{c}=4 n^{2} \sigma^{2} \sqrt{\pi k T / m}$; but the Knudson number in hypersonic flows is traditionally approximated by the ratio of the kinematic viscosity and the speed of sound, $N_{k}=\lambda / l \approx v / c$, to appear as the ratio of the local Mach and Reynolds number $M_{\infty} / \operatorname{Re}_{\infty}[1,2]$. The Knudson number will be reduced in the shock layer from the free-stream condition by the density ratio across the shock envelope. As long as the mean-free-path is less than the shock thickness, the flow field within the shock envelope is considered, at the most, in the rarefied gas domain, in which the velocity slip and temperature jump on the solid boundary may occur. A comprehensive pioneering effort in this research area by Tsein has been well documented [3]. Beyond this domain, the flow field belongs to the free-molecule flow regime that must be studied by the integro-differential Boltzmann equation.

The hypersonic small-disturbance theory is a nonlinear approximation because the disturbed velocity is not necessarily small in comparison with the free-stream speed of sound. The theory is appropriated for a slender body in hypersonic flows and inherently leads to the hypersonic similitude, a product of the local Mach number and a displacement thickness, $M \tau$, which is characterized by a value of unity for hypersonic flows $O(M \tau)=O(1)$. This similitude is logically unified with the Prandtl-Glauert similitude for general body shapes in linearized steady supersonic flow by the basic similitude parameter $\sqrt{M^{2}-1} \tau$. The fundamental idea of hypersonic similitude is derived from the observation that the magnitude of the perturbed cross-flow or the transverse velocity components is proportional to the product by the flow deflection angle and the freestream velocity as $\tau U_{\infty}$. Whereas, the disturbed stream-wise velocity component is proportional to the product by the disturbance rising to the square power, thus the unperturbed velocity has a magnitude of $\tau^{2} U_{\infty}$. The hypersonic similitude is derived formally by multiplying the stream-wise velocity component by the disturbance $\tau$ to reach the same order of magnitude of the transverse velocity counterpart. Simultaneously, a coordinate stretching transformation for the stream-wise coordinate is carried out. The resultant conservation laws based on Euler formulation show a second-order dependence of the stream-wise velocity; the resultant governing equations become the foundation for the law of cross-section or the equivalent principle [1, 4]. The physical concept can be summarized as that the flow as viewed in any transverse plane is independent of the flow in any other transverse plane. In essence, the hypersonic similitude reduces the number of independent variables for the inviscid hypersonic flow analyses.

The Newtonian theory was introduced to aerodynamics in the seventeenth century [5]. The impact law considers that the normal momentum transfer from gas particles is independent from the incidence of the body surface, thus there will be no transfer of tangential momentum. In gist, the Newtonian theory states that the component of impulse from an oncoming particle will contribute to the aerodynamic resistance proportional to the sine square of the incident angle. The condition of the Newtonian theory corresponds to a free molecule flow at the Mach number of infinite. This theory foreshadows the concepts of hypersonic similitude, Mach number independent principle, and the tangent wedge and tangent cone approximations.

Indirectly, the Newtonian theory also defines the aerodynamic shadow of any aerodynamic body moving with a high angle of incidence. Since the classic theory does not consider the existence of a bow shock or the impinged forebody with a longitudinal 
radius of curvature, some attempts have been made for improving the theory in practical applications. However, the most effective modification is simply by including the Rayleigh Pitot pressure formula to account for the static pressure recovered from a normal shock at the stagnation point [6]. An excellent affinity with numerical solutions by the solutions of the compressible Navier-Stokes equations has clearly demonstrated the predictive accuracy from the Mach number from 5 to 30 by the conceptually simple and elegant theory.

The Mach number independent principle provides a clear definition for hypersonic flow in a perfect gas by the asymptotic values of the Rankine-Hugonoit relationship. As the Mach number increases, the normal and oblique shock jump conditions become only a function of flow deflection angle and the specific heat ratio, as the consequence, the drag coefficients of a blunt body and a slender body approach a constant asymptote [7]. It also reveals that the hypersonic flow field of a blunt body reaches an invariant structure at a lower Mach number than that of a slender body. The unique aerodynamic behavior is displayed in Fig. 1, through which the distinctive features of hypersonic flow over arbitrary shape are quantitatively established.

An explosive pressure rises over the leading edge in hypersonic flow and encloses by a shock envelope with an inclining Mach angle with respect to the freestream. The blast wave theory also recognizes that the bow shock shape is relatively insensitive with respect to the wave generating body configuration. When the shock formation is viewed from the apex of the leading edge, the steady three-dimensional flow field structure in each transverse plane appears as concentric circles or ellipses. This observation is in a perfect accord to the law of plane section or the equivalent principle; the steady multi-dimension flow is now equivalent to an unsteady but a one-spatial-dimensionless flow field [8]. This insight and incisive intuition is the cornerstone of the blast wave analogue $[9,10]$. By the Lagrangian formulation, outstanding results have been obtained from the blast wave theory, and the accuracy by comparing with experimental observation has a varying different degree of accuracy. One must be mindful of the fact that the analogy only holds for the regions where the small perturbation equations are

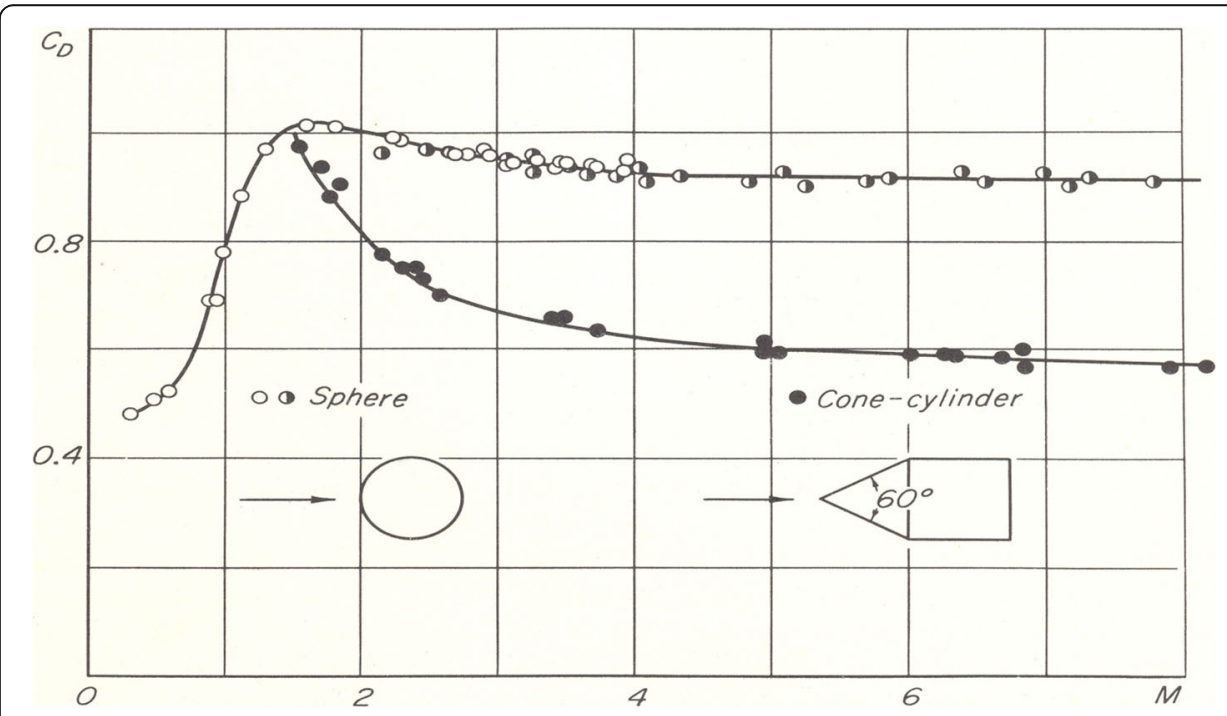

Fig. 1 Mach number independent principle of hypersonic flow 
Table 1 First excited quantum state and characteristic ion temperatures

\begin{tabular}{|c|c|c|c|}
\hline Electronic state & Ionization potential, ev & Energy level, ev & Characteristic $\Theta_{i,} \mathrm{k}$ \\
\hline $\begin{array}{ll}{ }^{1} D_{2} \\
\end{array}$ & 13.6 & 1.96 & 22,800 \\
\hline $\mathrm{O}_{2} \quad{ }^{1} \Delta_{g}$ & 12.2 & 0.98 & 11,300 \\
\hline$N^{2} D^{\circ}$ & 14.55 & 2.37 & 27,500 \\
\hline$N_{2}{ }^{3} \Sigma_{u}^{+}$ & 15.58 & 6.10 & 71,000 \\
\hline NO ${ }^{2} \Sigma^{-1}$ & 6.25 & 5.29 & 61,400 \\
\hline
\end{tabular}

valid. Thus the approximation will fail in the nose region. Nevertheless, the elegance of analytic results by the blast wave theory cannot be overlooked in that the general trend is confirmed from comparisons with experimental data [11].

The viscous-inviscid interaction over a sharp leading edge becomes much more pronounced at a hypersonic Mach number than supersonic flows. The Mach wave interaction or the pressure interaction arises from the fact that the growth of a thin boundary layer over an aerodynamic shape is no longer ignorable. The boundary layer originated from the leading edge displaces the surrounding external flow outward triggering a series of compression waves; the increased stream-wise pressure gradient transmits into the boundary inner layer. The local pressure gradient adjusts the shearlayer structure and reflects the changed displacement thickness to the external flow. The interactions persist until a dynamic balance is reached. Eventually the induced compression waves coalesced into a leading edge shock. A concise analysis was performed to show a strong $(\bar{X}>3.0)$ and a weak $(\bar{X}<3.0)$ Mach wave interaction that can be defined by an interaction parameter $\bar{X}=\sqrt{c} M_{\infty}^{3} / \sqrt{\mathrm{Re}_{x}}[1]$, and the analytic result is fully substantiated by experimental observation, which is exhibited in Fig. 2 [12]. The hypersonic flow over a blunt body also generates a vorticity distribution from the

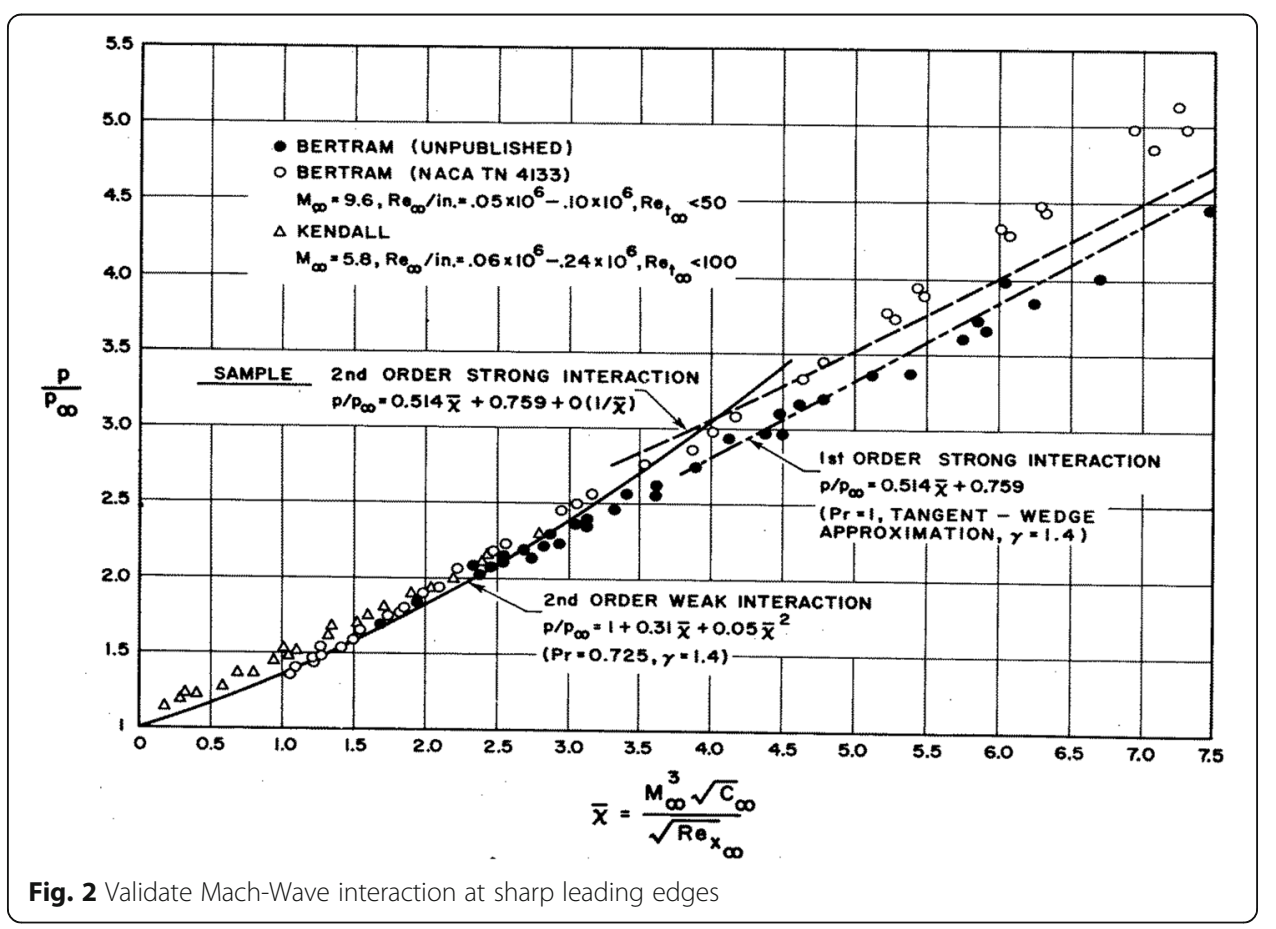


curved bow shockwave. The trailing vortices in slipping streams from the curved enveloping shock alter the outer boundary condition for the attached shear layer, and lead to the vorticity or streamline interaction. The net result of vorticity interaction increases the skin friction and heat transfer. This hypersonic leading edge interaction is described by the vorticity interaction parameter $\Omega=(\varsigma / \rho u)_{i n v} \int \rho d y$, and the symbol $\varsigma$ denotes the inviscid vorticity $\varsigma=\nabla \times u[1]$.

The accurate stagnation point heat transfer prediction is one of the most outstanding achievements by the classical viscous hypersonic flow theory. The compressible boundary-layer formulation of a chemical reaction and dissociating air is reduced to the incompressible Falkner-Skan equations by a combination of compressibility [13], coordinates transformations [14], and aerodynamic similarity parameters. The outer edge velocity of the thin shear layer from the stagnation point is realizing to be proportional to the distance from that point. This unique feature further reduces the equations into the self-similarity form, which yields accurate prediction for stagnation point heat transfer including chemical reaction for the dissociated air $[15,16]$. Figure 3 summarizes the remarkable and elegant achievement for the heat transfer distribution over blunt body to become a benchmark in hypersonic research.

The extremely abbreviated descriptions of hypersonic flow for the perfect gas only touch a very small fraction of the classical theories. It intends to bring out the exceptional and unique aerodynamic features of hypersonic flows, which is far from complete and comprehensive in describing the crucial knowledge based on the perfect gas approximation. It is astonishing to recognize that all these extraordinary theories are derived from the binary elastic collision model by the gas dynamic kinetics in which the atomic/molecular structure is not considered. Nevertheless, they are the timeless cornerstones and foundations for studying hypersonic flows [17]. Along this train of thoughts, it must be pointed out that the laminar-turbulent transition and turbulence that are completely governed by the classic gas kinetic theory remain as the only least understood phenomena in fluid dynamics. These chaotic vorticity interactions, rapid energy cascading and bifurcating physics rightfully belong to the last frontier of basic research [18].

The hypersonic flow exists mostly in a thermodynamic nonequilibrium state; the only correct nomenclature shall be the high-enthalpy gas dynamics. It arises from the fact that the internal structure of collision gaseous particles must be entered into consideration, in other words, the microscopic interactions between gases are inelastic collisions. From quantum mechanics, the internal degrees of freedom of atoms and molecules are no longer ignorable together with nonequilibrium chemical reactions among gas species. Whence the gas is ionized, the radiation as well as the electromagnetic force and energy transfer will always be presented to significantly alter the aerodynamic behavior. In essence, the high-enthalpy hypersonic flow constitutes a multiple scientific discipline. Under this circumstance, the classic hypersonic flow theories fall short on the accurate prediction for aerodynamic performance based on the perfect gas formulation.

Table 2 Designation of electron configurations of atom

\begin{tabular}{lllll}
\hline Shell & $K$ & $L$ & $M$ & $N$ \\
\hline Subshell/Element & $1 s$ & $2 s 2 p$ & $3 s$ 3p 3d & $4 s$ 4p 4d 4f \\
\hline
\end{tabular}




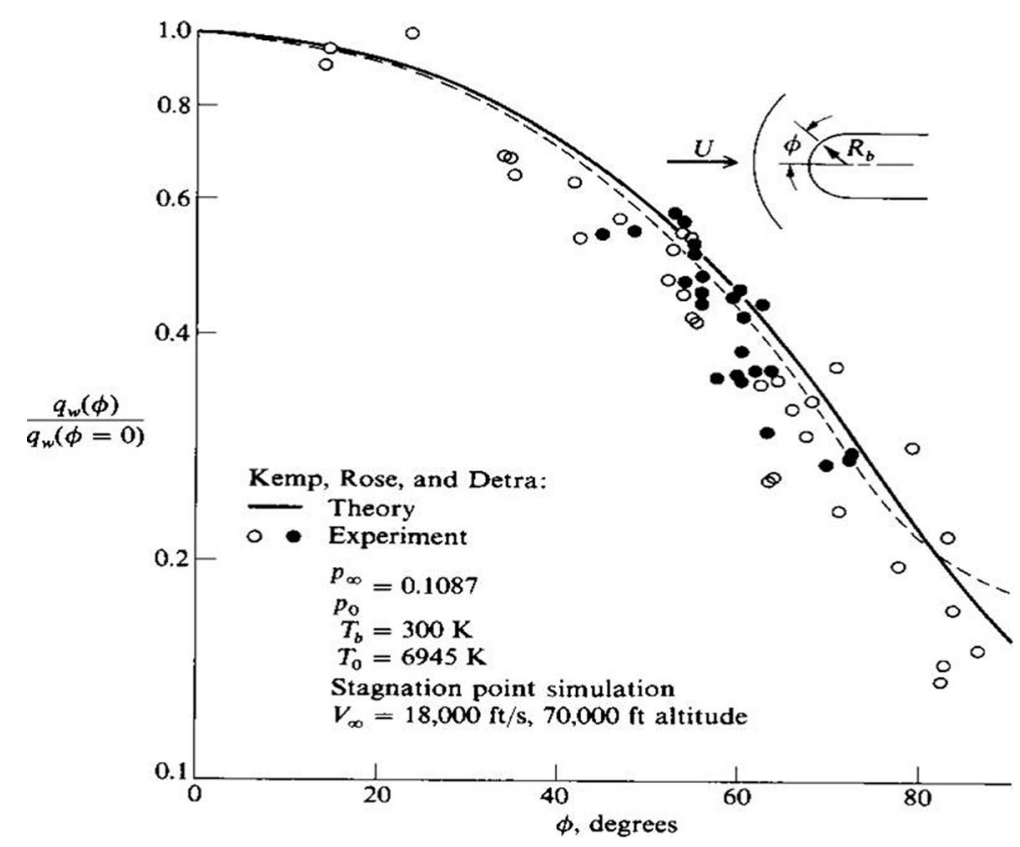

Fig. 3 Stagnation point heat transfer by compressible similar boundary-layer theory

The rapid progress in aerospace science for interplanetary explorations and sustained high-speed flights, demands a higher degree of physical fidelity for the high-enthalpy hypersonic flows. In order to meet the mandatory requirements, the subject of hypersonic flow must venture into an interdisciplinary domain by integrating the nonequilibrium chemical kinetics, ionization, quantum transition, radiative energy transfer, as well as, the electromagnetics with aerodynamics.

\section{Nonequilibrium chemical kinetics}

The kinetic-to-thermal energy conversion by a strong compressing shock must be accomplished in the thin shock layer, which is a fraction of the characteristic length of the leading-edge radius, thus the process is far from thermodynamic and chemical reaction equilibrium [19]. The environment of reentry is characterized by a speed around $10 \mathrm{~km} / \mathrm{s}$ or a Mach number about 30, and the air temperature within the bow shock envelope exceeds $20,000 \mathrm{k}$, which leads to a rapid thermal excitation including air ionization. The chemical composition of air starts to change at a temperature over $2500 \mathrm{k}$; the oxygen molecules are first dissociated together with a slight amount of nitric oxide formation. When the temperature arises over 4000 to $8000 \mathrm{k}$, the nitrogen will dissociate and all other molecular components begin to ionize, which transforms the air into an electrically conducting medium $[2,7,20]$. In the density range from one hundredth to 10 times of standard atmospheric condition, the high-temperature air mixture is widely considered to consist of 11 species: $\mathrm{O}, \mathrm{N}, \mathrm{O}_{2}, \mathrm{~N}_{2}, \mathrm{NO}, \mathrm{O}^{+}, \mathrm{N}^{+}, \mathrm{O}_{2}{ }^{+}$, $\mathrm{N}_{2}{ }^{+}, \mathrm{NO}^{+}$and $\mathrm{e}^{-}$. In fact, the chemical reactions are proceeding at a nonequilibrium finite rate for most hypersonic flows. Its impacts on thermodynamic properties of the gas medium are drastic, and the nonequilibrium chemical reactions become an increasingly important aspect in hypersonic flows. 
The molecular internal degrees of freedom are constituted by the translational, rotational, vibrational, and the electronic modes, and the electronic mode can still be split into dissociation and ionization excitations. In the analysis by the nonequilibrium chemical kinetics, the gas species at different internal modes are treated as distinctive chemical species. For molecular nitrogen, oxygen, and nitric monoxide, the characteristic temperatures of rotational excitation at the equilibrium states are 2.86, 2.07, and $2.42 \mathrm{k}$ respectively [2]. Therefore, the rotational mode for air molecules is fully exited in most circumstances and equilibrates with the translational mode; thus is analyzed collectively. From quantum mechanics, the energy states of all air internal citations have discrete spectrum, except the translational mode which packs so tightly that it is approximated as continuous.

In a closed chemical reacting system, the total mass of reactants in a chemical reaction is an invariant. According to the chemical kinetic theory, any complex chemical reaction can be broken down into multiple permissible single-step elementary reactions $[2,19]$. The law of mass action expressed a relationship between the species in numbers of moles;

$$
\Sigma v_{i}^{\prime} N_{i} \rightleftarrows \Sigma v_{i}^{\prime \prime} N_{i}
$$

The symbols $v_{i}^{\prime}$ and $v_{i}^{\prime \prime}$ are referred as the stoichiometric coefficients, and $N_{i}$ denotes the species concentration in mole per unit volume. Therefore the law of mass action may be viewed as a statement for conservation of total number atoms of reactants by the collective one-step elementary reactions processes [19]. The fundamental law establishes the base for modeling chemical reactions, and the rate of production and depletion of each species is determined by the mass conservation law. This law of mass action actually is an empirical formulation confirmed by numerous experimental observations, and it states that the rate of generation and depletion of a chemical species is proportional to the products of its concentrations rising to a power equal to the corresponding stoichiometric coefficients.

The first stage of any chemical reaction is the formation of an activated complex, or for the interacting molecules to have a sufficient amount of energy by collision and coalesce to form an activated complex. This complex can either decompose back to the individual reactants or precede forward into the reaction products. In a detailed potential energy analysis, it contains two types of interaction energy by the electrostatic Coulomb energy and the quantum mechanical exchange or the resonance energy. The process is rather complex. The strong temperature dependence of the chemical reaction rate merges to become the most striking feature. This phenomenon was recognized by Arrhenius and led to the Arrhenius law for the forward and backward reactions [2];

$$
k_{f / b, i}=A_{f / b, i} T^{n} \exp \left(-E_{f / b, i} / k T\right)
$$

The symbol $E_{f / b, i}$ is referred to as the Arrhenius activation energy, and the exponential term of the temperature is also known as the Boltzmann factor. The Arrhenius law simply states that only those reactants that possess energy greater than a certain potential barrier (activity energy) will lead to a reaction. The term $A_{f / b, i}$ is often called as the collision frequency to reflect its roots in statistical quantum mechanics, which is also known as the steric factor which depends on the relative orientation of colliding species 
for reaction to occur. Physically the steric factor can be interpreted as the ratio of the cross section for the reactive collisions to the total cross section [2, 20]. These reaction rate constants unfortunately are known to have a huge and varying degree of uncertainty.

It is customary to represent the deviation between theory and experiment by introducing the steric factor which depends on the collision models. In short, if the steric factor is less unity, then the orientation of the colliding molecules is significant, or a critical orientation of the energetic collisions has been included for the reaction to occur. In other words, it expresses the probability that the collision is in the correct geometric configuration to allow the reaction to take place $[19,20]$. When, the steric factor has a value greater than unity, then it needs more reaction collisions than the simple kinetic theory allows. In that situation, an alternative approach by the quantum theory becomes necessary. For this reason, it offers a great opportunity of basic research for chemical kinetics using high performance computational techniques [18].

The law of mass action establishes the foundation for modeling chemical reactions, and the rate of chemical reaction provides the source term to the mass continuity equation. The original Arrhenius formula recognizes the reaction constant has a strong temperature dependence to appear as [2];

$$
d \ln k_{i} / d t=\varepsilon_{i} / \kappa T^{2}
$$

The forward reaction rates of a finite-rate or nonequilibrium chemical reaction are;

$$
R_{f, i}=k_{f, i}(T) \Pi\left(\rho_{i} / M_{i}\right)^{v_{i}^{\prime}}
$$

Similarly, the rates of the backward chemical reaction are given as;

$$
R_{r, i}=k_{b, i}(T) \Pi\left(\rho_{i} / M_{i}\right)^{v_{i}^{\prime \prime}}
$$

where the symbol $M_{i}$ denotes the molecular weight of the chemical species $i$. The notations $k_{f, i}$ and $k_{b, i}$ are the so-called reaction rate constants which are functions of temperature and independent from the concentration of reacting species. At the present time, these reaction rate constants are determined by either from the experimental data or empirical means. The net rate of change for the $i$ species concentration, is the balance between an individual elementary one-step forward and backward reactions;

$$
R_{i}=k_{f, i}(T) \Pi\left(\rho_{i} / M_{i}\right)^{v_{i}^{\prime}}-k_{b, i} \Pi\left(\rho_{i} / M_{i}\right)^{v_{i}^{\prime \prime}}
$$

Through these finite rate chemical reactions, the reaction rate of each individual species in an nonequilibrium chemical reaction gas mixture is the sum of all permissible simple elementary chemical reactions;

$$
d w_{i} / d t=M_{i} \sum_{j=1}^{J}\left(v_{i, j}^{\prime \prime}-v_{i, j}^{\prime}\right)\left\{k_{f, j} \prod_{k=1}^{N_{k f}}\left(\rho_{k} / M_{k}\right)^{v_{k, j}^{\prime}-k_{b, j}} \prod_{k=1}^{N_{k b}}\left(\rho_{k} / M_{k}\right)^{v_{k, j}^{\prime \prime}}\right\}
$$

In order to complete the complex energy transfer process including all the internal degrees of freedom, the chemical kinetics and aerodynamic interactions are coupled by 
the source term for all chemical species. The rate of chemical reaction is also dependent on the energy cascading and redistributing among different quantum states of the same species, as well as, between internal degrees of freedom. Although the quantum jumps are instantaneous, the relaxation phenomenon between jumps may occur frequently, and the phenomenon is determined by the collision frequency and energy levels between quantum states.

The individual species conservation equations actually include the same species with different internal vibration and electronic degrees of excitations. The sum of these individual species conservation equations, Eq. (7) becomes the source term of the global continuity equation for the conservation law in a chemical reacting flow, which is the most fundamental law of Newtonian mechanics for which the mass and energy is not exchangeable like that in quantum mechanics. The species conservation equation including the species diffusion velocity $u_{i}$ becomes;

$$
\partial \rho_{i} / \partial t+\nabla \cdot\left[\rho_{i}\left(u+u_{i}\right)\right]=d w_{i} / d t
$$

The earliest chemical kinetics model of high-temperature air is developed by Kang and Dunn [21] which composes of twelve species and sixty-four elementary chemical reactions, and later simplifies to eleven and twenty-six reaction. In this model, all internal degrees of excitation are assumed to retain at a thermal equilibrium condition. The rates constants for the recombination of charged species are measured from the application conditions. For this reason, the reaction rate coefficients for the charge transfer and ion-molecule reactions are uncertain, because the reaction rates are substantially influenced by the nonequilibrium process. The most widely adopted OlynickChen-Tauber chemical kinetics model [22] actually is a continuing improvement from the Park's original effort and extends its application to include ablation phenomena [23]. The reaction rate constants are tabulated with the approximated temperatures, assuming the Maxwell distribution function between microscopic and macroscopic states is still valid, and applied to simulate finite-rate chemical reactions [19].

The computational simulations for nonequilibrium chemical reactions have been verified with the flight data from a sample return probe RAM-C-II (Radio Attenuation Measurement), which is basically a blunt-nose conic body with a nose radius of 15.24 $\mathrm{cm}$, a semi-cone angle of nine degrees, and an overall length of $1.2954 \mathrm{~m}$. The data were collected from the altitude range from 85.3 to $53.3 \mathrm{~km}$, and at the approximated reentry velocity of $7.62 \mathrm{~km} / \mathrm{s}$. In Fig. 4, the species number density (number per cubic centimeter) of an eleven-species air mixture is displayed along the shoulder line in the shock layer over the probe at different stages of the reentry trajectory $(81,71$, and 51 $\mathrm{km})$. The oxygen dissociation is followed by nitro oxide and the dissociated nitrogen. The ionization process becomes more noticeable only in the later stages of the earth reentry trajectory, and the globally electrical neutrality of the positively charged ions and electrons is also revealed [19].

In order to demonstrate the effects of chemical reactions to the thermodynamic state of hypersonic flow, the translational temperatures distributions of a perfect gas and a chemical reacting air in equilibrium and nonequilibrium conditions are presented together in Fig. 5. All simulations are conducted for an axisymmetric hemispherical cylinder at the free stream Mach and Reynolds numbers of 27.89 and $1.38 \times 10^{4}$ respectively, and the air temperature is assigned a value of $198.6 \mathrm{k}$ [18]. A huge 


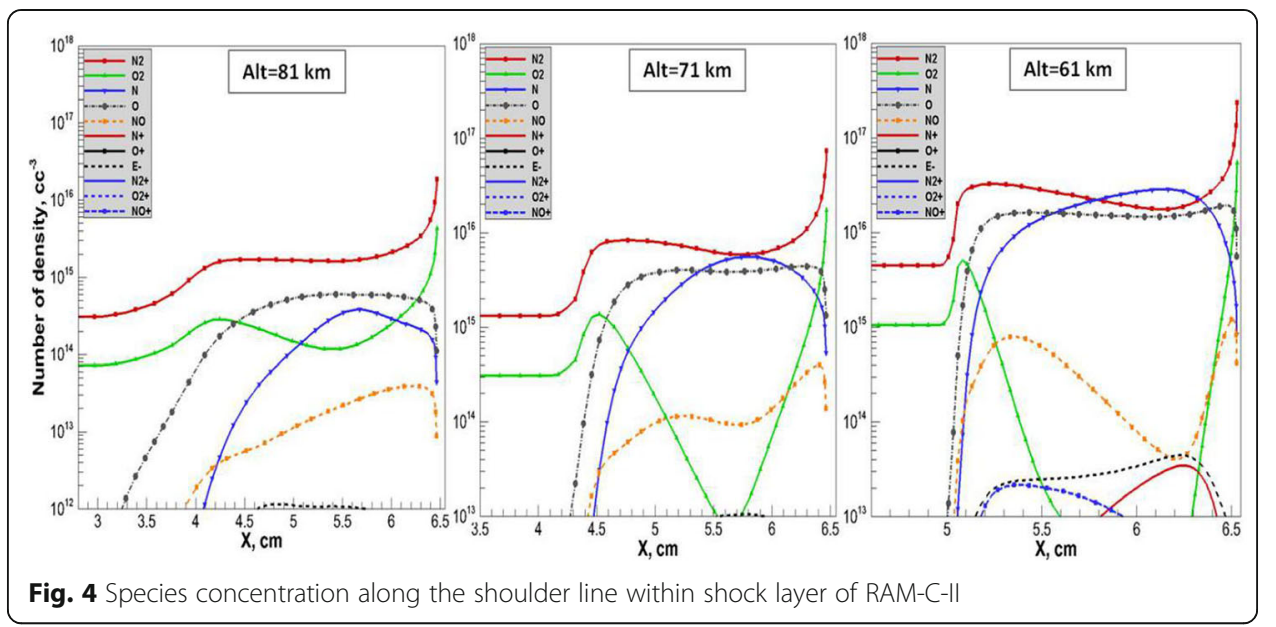

difference between the perfect gas and chemical reacting gas is clearly discernible under the hypersonic flow condition. It has been estimated at an ambient temperature of $1000 \mathrm{k}$, the vibrational mode requires between 1000 and 10,000 collisions to reach the equilibrium condition with translational mode $[2,19]$. Under this condition, the energy exchange between air molecules has a greater probability than the translation-vibration transition within the same molecule. Therefore it would be unrealistic for the chemical reactions including quantum jumps to be in an equilibrium state [18, 19].

In Fig. 5, the nonequilibrium temperature is an approximation by assuming the Maxwell distribution still holds between microscopic and macroscopic states. The computational results indicate the perfect gas formulation yields the highest translational temperature over $27,500.00 \mathrm{k}$, because all kinetic energy of the oncoming air is unable to convert into other internal degrees of freedom. The peak translational temperature of a nonequilibrium chemical reaction condition attains a value of $20,787.46 \mathrm{k}$, whereas the counter part of the equilibrium computation yields a much lower value of 5855.44 $\mathrm{k}$ [24]. From all reentry flight records at a similar condition, the translational temperature by equilibrium condition seems to be much lower than all recorded data

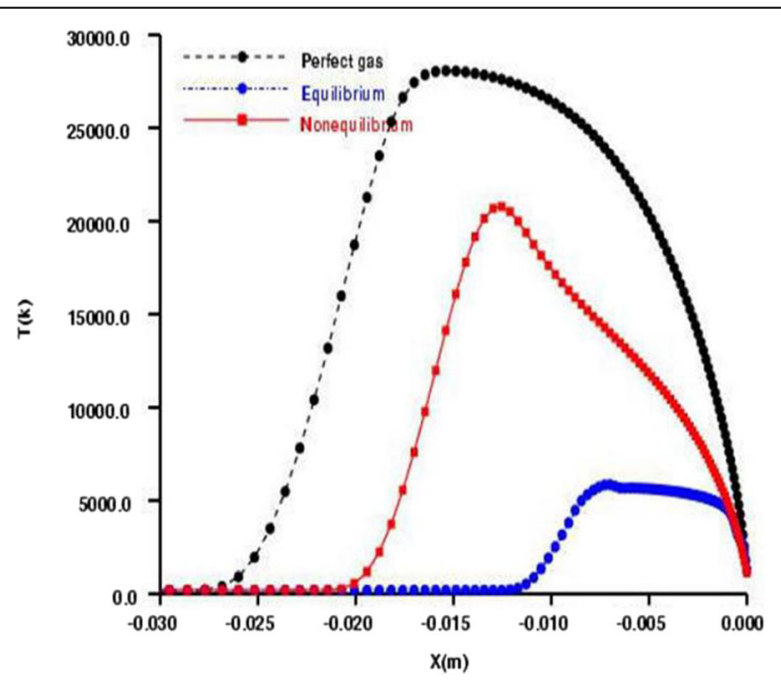

Fig. 5 Translational temperatures distributions along stagnation streamline 
$[19,23]$. The bow shock standoff distance is also reduced significantly from the perfect gas model. The decreased magnitude is mostly contributed by the altered air composition, which is directly related to the density ratio across the normal shock. Unfortunately, these computational simulations are generated by the shock capture technique without the aid of a suitable flux limiter, thereby numerical results fail to duplicate a sharp definition of the shock front [24].

Figure 6 presents the calculated species number densities in cubic centimeter along the stagnation streamline within the shock layer by the nonequilibrium chemical reaction formulation [25]. The simulated results duplicate a reentry condition of the RAM-C-II probe at the altitude of $62 \mathrm{~km}$. The flow field is characterized by a Mach number of 23.9 and the Reynolds number of $1.95 \times 10^{4}$. The shock standoff distance is defined by the temperature jump, to have a value of $0.79 \mathrm{~cm}$, and agrees well with all previous simulations [19, 22, 23]. The displayed results consist of 11 molecular, atomic, and ionized species, $\mathrm{N}_{2}, \mathrm{O}_{2}, \mathrm{NO}, \mathrm{N}$, $\mathrm{O}, \mathrm{N}^{+}, \mathrm{O}^{+}, \mathrm{N}_{2}{ }^{+}, \mathrm{O}_{2}{ }^{+}, \mathrm{NO}^{+}$, and $\mathrm{e}^{-}$. The species number densities over the value range from $10^{12}$ to $10^{18}$ are included. As an interesting observation from Fig. 6 , the highest number density of the electron is $4.2 \times 10^{14}$, which is merely a tracing amount of the ionized air. A detailed numerical resolution refinement has been carried out by increasing the grid number point density by a factor of 6.72 times. The affinity among different numerical results is remarkable, which even extends to the trace elements to exhibit that the numerical result is grid independent. The numerical results are also partially validated by plasma physics in that the sum of positively charged ionized species number densities equals to the number density of the free electrons to reflect the intrinsic globally neutral property of plasma.

\section{3 lonization}

The ionization process of atoms or molecules is initiated by the energized electrons that leave a stable orbit revolving around the nucleus and become free-moving charges. The typical ionization potential of an atom or a molecule for most air mixture is on the order of $10 \mathrm{eV}\left(1 \mathrm{ev}=1.602 \times 10^{-19} \mathrm{erg}\right)$. Some typical values are included in Table

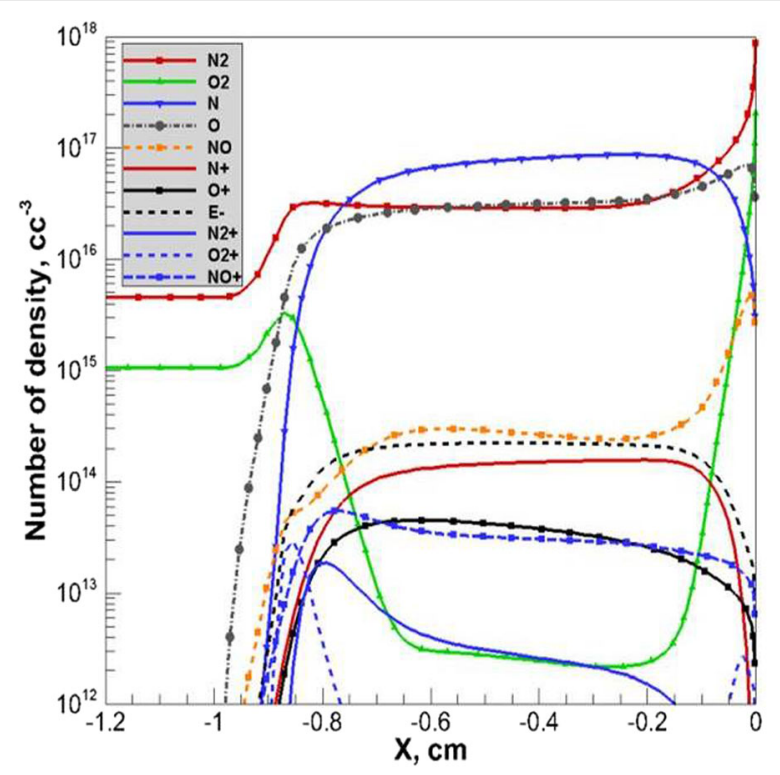

Fig. 6 Species concentration in shock layer of RAM-C-II Probe 
1. The excited state also decays spontaneously to a lower energy state by emitting photons on a time scale around $10 \mathrm{~ns}$ or $10^{-8}$ seconds [26]. Therefore the ionization of gas always exists in a transient state in time and space, and requires to be continuously supplemented by an external energy source. In general, the required energy for the highly excited states of atom and molecule must be provided by thermal, chemical, electrical, and radiative resources or by a combination of some, even by all mechanisms.

There are a total of eight basic ionization mechanisms: inelastic collision, electron impact, radiative interaction, charge exchange, dissociative recombination, dielectric recombination, and electron attachment [26, 27]. The dominant mechanism in hypersonic flows is the inelastic collisions, and the electron impact or electrical extraction is adopted by the electric propulsion system and for flow control. These processes can be described by the elementary chemical reactions;

$$
A+B \rightleftarrows A^{+}+e^{-}+B
$$

and

$$
\begin{aligned}
& E(A) \rightleftarrows A^{+}+e^{-} \\
& e^{-}+A \rightleftarrows A^{+}+2 e^{-}
\end{aligned}
$$

The ionized air in hypersonic flows is a quantum phenomenon through the altered internal structure of an atom or a molecule by thermal excitations or elastic collisions. The discrete quantum states of atom are obtained by solving the Schrödinger wave equation $[2,19]$.

$$
\left(h^{2} / 2\right) m \nabla^{2} \phi-V(r) \phi+i h \partial \phi / \partial t=0 ; \quad D \phi=\lambda \phi
$$

The quantum numbers describe that the energy state of an atom can be briefly summarized by following a single electron or multiple electrons. The dynamic states of an atom are always under the influence of a magnetic field in rotational and spinning motions of electrons, and are designated by quantum numbers of $n, l, m$, and $s$. The principal quantum number $n$ is a discrete integer, which is originated from the Bohr orbits in a radial coordinate. The quantum number $l$, denotes the azimuthal quantum number related to angular momentum. The magnetic quantum number $m$, is related to orientations of electrons under the influence of an external magnetic field, and the spin quantum number is designated as $s$. In principle, one can construct an eigenfunction corresponding to the energy states in which a definite set of orbits is occupied, but the energy state cannot be specified for an electron that occupies a particular orbit, because the orbit shall be described as the shells or electron clouds about the nuclei $[2,28]$.

The Pauli Exclusion Principle specifies the basic rule of permissible quantum states, namely that no two electrons of the same atom can have the same four quanta numbers. Four different elements of electronic orbits or the subshells exist for each set of energy levels or shells $K, L, M$, and $N$; they are designated as $s, p, d$, and $f$. And the electron configurations of gaseous atoms are given as tabulated in Table 2.

The slightly different energy levels in a quantum state are indicated by the upper left index 1,2 or 3 on a subshell or an element as ${ }^{2} S,{ }^{2} P,{ }^{1} D \ldots . .$. , and the quantum number $l$ for doublets terms is indicated as ${ }^{2} S_{1 / 2},{ }^{2} P_{1 / 2},{ }^{2} P_{3 / 2},{ }^{2} D_{5 / 2}$. For examples, the lowest orbit of one electron of hydrogen atom is in the $1 \mathrm{~s}$ orbit and the ground state is ${ }^{2} S_{1 / 2}$. The 
lowest electron configuration of nitrogen atom has three electrons which give the symbols of ${ }^{4} S,{ }^{2} D,{ }^{2} P$, and the ground state is ${ }^{4} S_{3 / 2}$. Therefore, the invert triplet ground states of oxygen are given as ${ }^{3} P_{2},{ }^{3} P_{2},{ }^{3} P_{0}[2,19,28]$.

The quantum number description for electronic configurations of a molecule is similar to that of an atom. Usually, the quantum numbers of a molecule consist of three groups. The first group is defined by the Born-Oppenheimer approximation for which the nuclei are held stationary and only the electronic motions are considered [2]. Second, only a single quantum number is used to define the vibration state of the nuclei. Third, the finer details of the electronic motion may not be separable and to be considered together as the ${ }^{1} \Sigma$ states. It is often defined by the five quantum numbers to specify the weak spin-orbit interaction as $\Lambda, S, v, J, K$. The first quantum number describes the electron orbital angular momentum, and the symbol $S$ designates the total spin angular momenta of electrons. The nuclei vibration quantum is given as $v$; the rotational quantum number is denoted as $J$. Finally, the quantum number $K$ is oft adopted for the compound orbital angular momentum of electrons and nuclei $[2,19]$.

The energy levels for rotational and vibrational excitations are derived from a diatomic molecule model by an idealized rigid rotor and simple harmonic oscillator without coupling in between;

$$
\begin{aligned}
& e_{r}=J(J+1) h^{2} / 8 \pi^{2} I, \quad J=0,1,2, \cdots \\
& e_{v}=(v+1 / 2) h v, \quad v=0,1,2, \cdots
\end{aligned}
$$

where $h$ is the Planck constant $\left(h=6.6261 \times 10^{-27} \mathrm{erg} \cdot s\right)$. Strictly speaking, the above results are valid only for the ${ }^{1} \Sigma$ molecular state.

The Maxwell distribution under collision equilibrium state is the sole link between individual particle dynamics to the thermodynamic variables. In fact, the distribution function establishes a definable relationship between the properties of microscopic and macroscopic states under the equilibrium state. The sum of the most probable states is the partition function $Z$, which distributes the energy of all particles among all the energy groups. In essence, it defines the most probable state for the existence and independence between internal modes across the microscopic and macroscopic description of gas. The independent variables of the partition function are the specific volume and temperature of a thermodynamic system;

$$
Z(V, T)=\sum_{i} g_{i} \exp \left(-e_{i} / k T\right)
$$

where the notation $g_{i}$ is the so-called degeneracy, which provides the detail formation for the internal atom/molecule structure [2, 20]. From this classification of the internal degrees of excitation in translational, rotational, vibrational, and electronic modes, the partition function of a molecule is the product of all or the factorization of its partition functions.

$$
Z(V, T)=Z(V, T)_{t} Z(V, T)_{r} Z(V, T)_{v} Z(V, T)_{e}
$$

For an atom, the partition function only has the translational and electronic modes.

$$
Z(V, T)=Z(V, T)_{t} Z(V, T)_{e}
$$


It is important to know that the factorization property of the partition functions is only valid so long as each energy mode can be assigned an energy level which is independent from the other energy modes. For hypersonic flows of the most circumstances, the coupling between modes is ignored.

From quantum mechanics, the molecular partition functions of each internal degree of freedom are obtained by integrating quantum solutions over all permissible quantum spectrum of each mode [2]. For an example, a simple harmonic model has been adopted for the vibration excitation by assigning the degeneracy of unity; the partition function of vibrational excitation after integrating over the entire range of quantum numbers can be expressed as $Z_{v}=\int g_{i} e^{-e_{i} / k T} d i \approx \Sigma e^{-i(v+1 / 2) h / k T}=[2 \sinh (h v / k T)]^{-1} ; 1$ $<v<\infty$. Similarly, individual partition functions in equilibrium conditions for the translational, rotational, vibrational by different models can be given, except the electronic excitation due to its multiply ground states remains as the sum of unspecified quantum solutions [2, 20, 29];

$$
\begin{aligned}
& Z_{t}=\left(2 \pi m k T / h^{2}\right)^{3 / 2} V \\
& Z_{r}=\left(8 \pi^{2} I k T / \sigma h^{2}\right), \sigma \text { symmetric factor } \\
& Z_{v}=1 /[2 \sinh (h v / 2 k T)], \text { simple harmonic oscillator } \\
& Z_{v}=\exp (-\Theta / 2 T)\left\{\left[1-\exp -\left(v_{\max }-1\right)\left(\Theta_{v} / T\right)\right] /\left[1-\exp \left(\Theta_{v} / T\right)\right]\right\} \text { Anharmonic oscillator } \\
& Z_{e}=g_{0} \exp \left(-e_{0} / k T\right)+g_{1} \exp \left(-e_{1} / k T\right)+g_{2} \exp \left(-e_{2} / k T\right)+\cdots
\end{aligned}
$$

All thermodynamic properties: the internal energy, pressure, and entropy of a system in the equilibrium state can be easily evaluated from the formulations of the statistics thermodynamics $[2,19]$. It may be noticed that only the translational mode can contribute to the partial pressure of a species.

$$
\begin{aligned}
& E=n \kappa T^{2}(\partial \ln Z / \partial T)_{\nu} \\
& P=n \kappa T(\partial \ln Z / \partial V)_{t} \\
& S=n \kappa\left\{[\ln (Z / N)+1]+T(\partial \ln Z / \partial T)_{\nu}\right\}
\end{aligned}
$$

Figure 7 depicts a schematic for the spectra of the four internal degrees of freedom in a molecule to highlight the discrete energy increments between quantum states. The energy differences between quanta become significant for rotational, vibrational and electronic excitations. The quantum jump is known to be accomplished either by a single level transition, known as the ladder climbing transition, or by a multiple-levels transition to be known as the big bang process. Even though the quantum jump is instantaneous, at times, very short but a finite time period may be elapsed between collisions to accumulate a sufficient amount of energy to proceed. The waiting period leads to the relaxation phenomenon for quantum transition $[2,20,30]$.

As an illustration for quantum transition, Fig. 7 also presents all potential energy curves for $\mathrm{O}_{2}$ and $\mathrm{NO}$ molecule that are developed from the spectroscopic data [30]. The horizontal lines indicate the vibrational energy levels for each electronic quantum state. According to the Frank-Condon principle, the spontaneous transition between quanta is most likely when the nuclei occupy the extreme locations, or intersection points. The rearrangement of the electron shell is estimated around $10^{-16} \mathrm{~s}$ [30]. From the classic viewpoint, the potential energy of the vibrational internal degree of freedom depends on the internuclear distance. Thus, the minimum point of the potential energy is the location of the equal attraction and repulsion force between molecules. This 


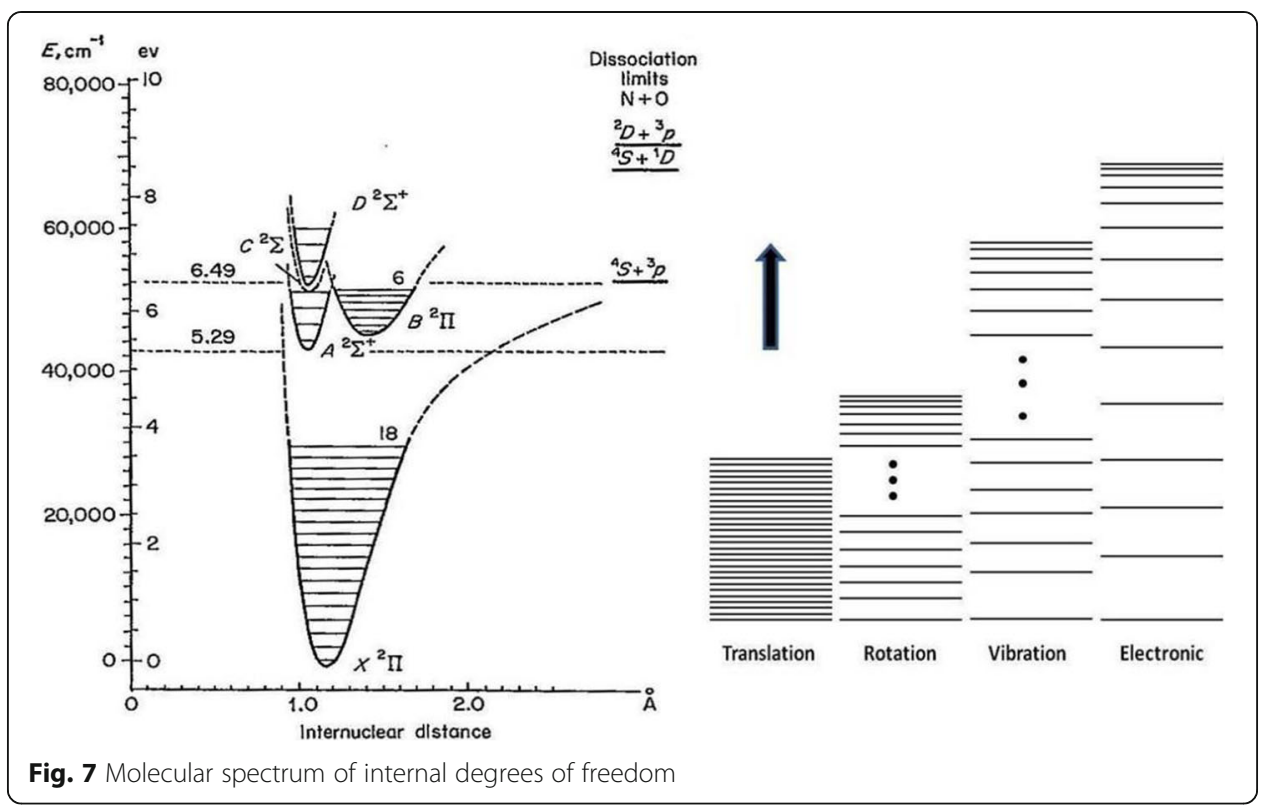

location is also known as the saddle point or the critical point of the zero-flux energy surface $[2,19]$.

In Fig. 8, the computational electron number density distributions and the accompanied data are presented along the RAM-C-II probe surface. On the right-hand-side of the figure, the flight data from the probe are recorded at a reentry altitude of $71 \mathrm{~km}$, the hypersonic Mach number decreases to 25.9, and the Reynolds number increases to $6.28 \times 10^{3}[25]$. The shock standoff distance from the data record indicates an estimated value of $0.7 \mathrm{~cm}$. These results of two limiting equilibrium and nonequilibrium chemical reactions are appended for purpose of comparison with the data. It is revealed that the nonequilibrium chemical reaction transits from the stagnation region toward downstream and approaches a nearly equilibrium chemical reacting condition. This behavior

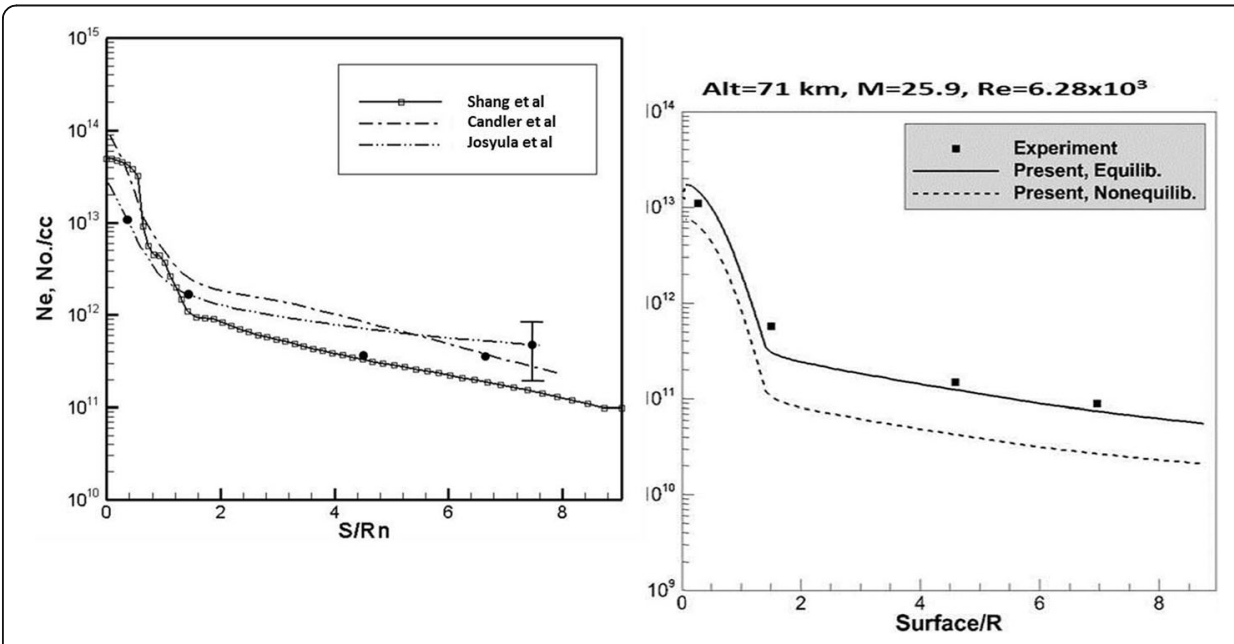

Fig. 8 Validating electron number density with flight data from RAM-C-II probe 
is anticipated because the numbers of collision between species increase as the gas mixture moves downstream from the bow shock wave.

Three independent computational simulations by different chemical-physics models are displayed in the left-hand-side of Fig. 8, as a specific comparison with the flight data. These numerical results show significant deviations from each other to reflect the state of progress over the past thirty years $[19,29]$. The flight data were collected by two different techniques; most of it by a reflectometer, the last checking point is recorded by an electrostatic probe. All these computational results are laid within the estimated error band of $3.0 \times 10^{11}$ to $1.2 \times 10^{12}$ per cubic centimeters, due to probe motion and accuracy of different measurement techniques.

All the ionization processes involve quantum chemical-physics and nonequilibrium chemical kinetics. In the nonequilibrium chemical computational process, the ionized species in different internal degrees of freedom are treated as the independent reactants and products. When the detailed composition of the ionized gas mixture is not of a concern, such as the ionized gas adopted for flow control, only the number densities of electrons and positively charged ions are needed. Then the physics-based modeling is built on the Townsend similarity law for ionization. The depletion by recombination, electron attachment, and detachment processes are included from the ionized gas kinetics [26, 27, 29].

Figure 9 presents a side-by-side direct current discharge (DCD) for flow control at a hypersonic rarefied gas leading edge of a flat plate [19]. The computational simulation and the photograph are produced under identical conditions; at a Mach number of 5.0 and an ambient pressure of 5.0 Torr (millimeter of $\mathrm{Hg}$ ). Each electrode has a length of $0.5 \mathrm{~cm}$ and a width of $0.25 \mathrm{~cm}$, and the gap between cathode (on the left) and the anode is $1.5 \mathrm{~cm}$. The discharge is maintained by an electric potential of $439.0 \mathrm{~V}$ with a discharge current of $5.2 \mathrm{~mA}$. The experimental observation shows that the electric field intensifies at the sharp edge of the electrodes, and the local electron number density has a maximum value of $1.7 \times 10^{10} / \mathrm{cm}^{3}$. From the contour presentation, the cathode layer can be clearly discerned, and the plasma sheath is clearly displayed in the cathode layer. Meanwhile, the Joule heating is concentrated along the inner edge of cathode layer closest to the anode, at a total applied direct current electric power of $21.52 \mathrm{~J} / \mathrm{cm}^{2} \mathrm{~s}$; the Joule heating of the glow discharge at the pressure of 5 Torr is estimated to be $1.30 \mathrm{~J} /$ $\mathrm{cm}^{2} \mathrm{~s}$ [19]. The Joule heating abruptly increases the local displacement thickness and leads to a viscous-inviscid interaction. In turn, the induced compression waves coalesce into an enforced oblique shock at the leading edge for flow control.

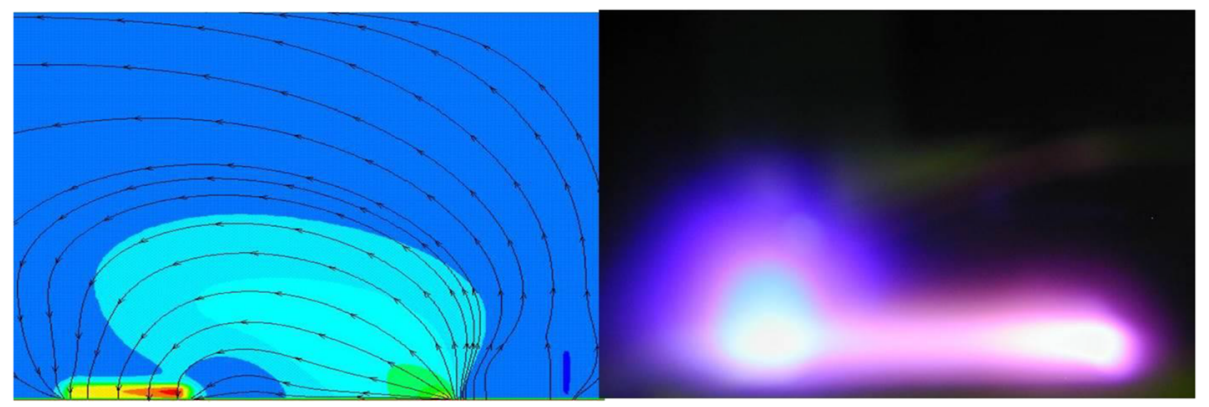

Fig. 9 Direct current discharge over a side-by-side electrode arrangement for flow control 


\section{Electromagnetics of ionized air}

Even the ionized air is weekly partially ionized plasma within the hypersonic shock layer, and yet the flow medium becomes an electrical conductor. The electrostatic and Lorentz forces, magnetic pressure, Joule heating, and radiative energy are presented, together with the communication blackout phenomenon from the dissipation and reflection of microwave propagating through the plasma. In order to achieve an acceptable physical fidelity for hypersonic flows, the governing physics now must include the electromagnetic discipline.

Two fundamental and intrinsic characteristics of the ionized gas or plasma are the Debye shielding length and the plasma frequency [31]. The unique property of plasma is the tendency maintaining electric neutrality; this particular condition requires an enormously large electrostatic force between an electron and ion. In a homogeneous field and isotropic medium, the electric field intensity $E$ is proportional to the gradient of the electric potential $E=-\nabla \phi$, and the Debye shielding length can be determined either by the dynamics of charged particles or by the balance of electrostatic and thermodynamic variables to get;

$$
\lambda_{d}=\left(\varepsilon \kappa T / e^{2} n\right)^{1 / 2}
$$

where the symbols $\varepsilon$, e, and $n$ denote the electric permittivity $\left(\varepsilon=8.854 \times 10^{-12}\right.$ Farad / $m)$, the elementary electric charge $\left(e=1.022 \times 10^{-19}\right.$ Coulomb), and the charge particle number density respectively.

The Debye shielding length represents the finest characteristic length scale of plasma, and is on the same order of magnitude of the electron mean free path, $10^{-6}-10^{-7} \mathrm{~m}$ $[29,31]$. At a temperature of $2500 \mathrm{k}$ and a charge number density of $10^{20} / \mathrm{m}^{3}$, the Debye length is $1.14 \times 10^{-7} \mathrm{~m}$. However, the charge neutrality of plasma does not always prevail in the immediate adjacent region to a solid surface, particularly around electrodes, to become the so-called plasma sheath. A major contributing factor to this behavior is the chemical recombination mechanisms by neutralizing the charges of ionized gas when striking the surface. The electric potential drop in the sheath has been estimated as $\phi_{s h}=-(\kappa T / 4 e) \ln \left(m_{i} / m_{e}\right)$; it may be recalled that the ratio of unit masses between ion and electron has a magnitude close to 2000 [32].

When the globally neutral condition is perturbed, the charged particles will always return to its original equilibrium state. However, the inertia of electrons always unavoidably overshoots, and the subsequent oscillation leads to the plasma frequency. The fundamental frequency is determined by the electron momentum equation as $[19,26]$.

$$
\omega_{p}=\sqrt{n e^{2} / m \varepsilon}
$$

A typical plasma frequency by the electron impact ionization has a value around $8.7 \times 10^{9} \mathrm{~Hz}$.

One of the most dramatic manifestations of plasma is the properties of an electromagnetic (EM) wave, which is constituted with the longitudinal sonic and the magnetic transverse wave components such as the Alfven wave [33]. The intensity of a propagating microwave wave always attenuates as it interacts with the electromagnetic field in an ionized gas or plasma. When an incident microwave frequency is lower than the 
plasma frequency, a complete reflection of the incident wave occurs at the medium interface, resulting in the well-known communication blackout [19].

In plasma, the electrostatic force between two singly charged particles is described by the Coulomb's law [34]. The force between charged particles is collinear along the unit space vector between two charges separated by a distance $r_{i j}$. The principle of superposition applies for multiple point charges; the electric field intensity becomes the sum of all elementary charges;

$$
E=(1 / 4 \pi \varepsilon) \Sigma\left(q_{i} / r_{i j}\right)
$$

The free electric charge in motion produces a conductive electric current. In metallic conductors, the charge is carried by electrons; in liquid conductors, such as electrolytes, the charge is carried by both positive and negative ions. The electric field compels the free charges into a continuous motion and results in an electric current which can be defined in term of the electric flux vector per unit area by the Ohm's law [19, 34];

$$
J=n q u=\left(n q^{2} / m v_{e}\right) E
$$

The conductive current density is different from the convective and displacement current. The convective current flows through an isolating medium and is exemplified by an electron beam within a vacuum tube, which does not involve electrically conducting medium and consequently does not obey Ohm's law [27]. The displacement current arises from the time-varying electric field and is introduced by Maxwell to account for its presence in the vacuum by a changing electric field in time. Without the displacement current, the electromagnetic wave propagation would not be possible [34].

The magnetic field can be generated either by magnets or by an electric current, and the orientation of the induced magnetic field is defined by the right-hand-rule [34]. The Biot-Savart law gives the differential magnetic field intensity $H$ or the magnetic flux density $B(B=\mu H)[19,27]$. The relationship between them is the magnetic permeability with the symbol of $\mu\left(1.257 \times 10^{-5}\right.$ Henry $\left./ m\right)$. The magnetic flux density is given as;

$$
B=(\mu / 4 \pi) \iiint\left(J \times l / r^{2}\right) d V
$$

The individual charged particles have the $E \times B$ drift and diffusion velocities in an electromagnetic field between charged particles collisions. The collisions and dissipation processes become the electrical resistive phenomenon and lead to the Joule heating. According to the Ohm' law, the conductive current density $J$ is related to the applied electromagnetic field by a bulk electric conductivity $\sigma[20,26]$;

$$
\begin{aligned}
& J=\sigma(E+u \times B) \\
& \sigma=n q^{2} / m v_{e}
\end{aligned}
$$

The electric conductivity is the reciprocal of the electric resistance, and has a SI unit of ohm-meter. In general, it is a tensor and only in the absence of magnetic field and in an isotropic medium it degenerates into a scalar.

In an electromagnetic field, the interaction of the electric field with the free charge density of the medium generates the electrostatic force $F=\rho_{e} E$. The interaction with an externally applied magnetic field by an electric current produces a force $F=J \times B$ within the plasma, and known as the Lorentz force or acceleration. The current density is 
directly related to charge particle motion, thus the total electromagnetic force is $F=$ $q(E+u \times B)$. Since a moving charge $q$ in a steady external applied magnetic field $B$ will be pushed by the Lorentz force normal to it, the velocity component parallel to $B$ will not be affected. But the velocity component normal to the magnetic field must follow a circular trajectory and execute a spiral motion. The gyro angular velocity is balanced by the centrifugal force, thus the Lorentz acceleration has a cyclotron or Larmor frequency, $\omega_{b}=e B / m$. In three-dimensional space, the charge particle trajectory in an electromagnetic field becomes prolate cycloid or curtate cycloid with loops [27].

The externally applied magnetic field also generates the Maxwell stress tensor like that of the velocity dyadic tensor in gas; the normal stress component, $B \cdot B / 2 \mu$ is the magnetic pressure similar to the hydrodynamic pressure. In theory, the transverse magnetic component can modify the fundamental Rankine-Hugoniot relationship for oblique shock jumps $[19,35]$, but must require a very strong magnetic field strength which can only be provided through the super conductivity technology. The externally applied magnetic field also produces the Hall effects by the gyrating acceleration of charged particles in plasma [36]. Due to the disparity of unit mass of an electron and ion, a transverse current $J_{h}=$ $n_{e} e(E \times B)$ is generated by the different curvature of their trajectories between them. The Hall current therefore has been shown a dependence on the ratio of the electron cyclotron frequency to the electron collision frequency [19,29].

The trajectory of charged particles motion in a Hall-effect ion thruster for space flights or satellite station keeping is depicted by a direct simulation Monte Carlo (DSMC) solution in Fig. 10 [37]. The magnetic field is generated by magnets installed outside of the thruster chamber; the maximum magnetic flux density is $600 \mathrm{G}-\mathrm{cm}$ at the magnetic poles. The electron trajectory displays the typical and characteristic pattern in the presence of an externally applied magnetic field. The magnetic field lines constrain and dictate the electron movements by the Lorentz force. All electrons accelerate linearly by the electrostatic force; meanwhile execute a gyrating motion perpendicular to the magnetic field flux by the Lorentz force. The trajectory of the charge particles motion is the well-known curtate cycloid with loops.

Figure 11 demonstrates the existence of the magnetic pressure generated by an externally applied magnetic field. The magnetic flux density is produced by an electric coil located coaxially in a hemispherical cylinder that immerses in an ionized air shock tube. The maximum magnetic field strength is 4.0 Tesla and the ionized air is estimated to have an electric conductivity in the range of $28-55 \mathrm{mho} / \mathrm{m}$. The activated magnetic field displaces the bow shock wave upstream through the added magnetic pressure which decreases the air density in the shock layer [19]. However, the quantification for the increased standoff distance from the experimental observation is not ascertained because of the optical distortion by the emitting discharging glow [38].

All electromagnetic phenomena are governed by the Maxwell equations in the time and space domain, which were established in 1873 as the foundation of electromagnetic theory [39]. The intrinsic rules of nature are beyond any doubt and couple many other scientific disciplines especially with radiation, plasmadynamics and magnetohydrodynamics (MHD). The Maxwell equations in the time domain consist of a firstorder divergence-curl system which is classified as the hyperbolic partial differential equations. In practical aerospace engineering applications, the system of equations has been widely applied in plasma generation, microwave energy transfer, as well as, radar 


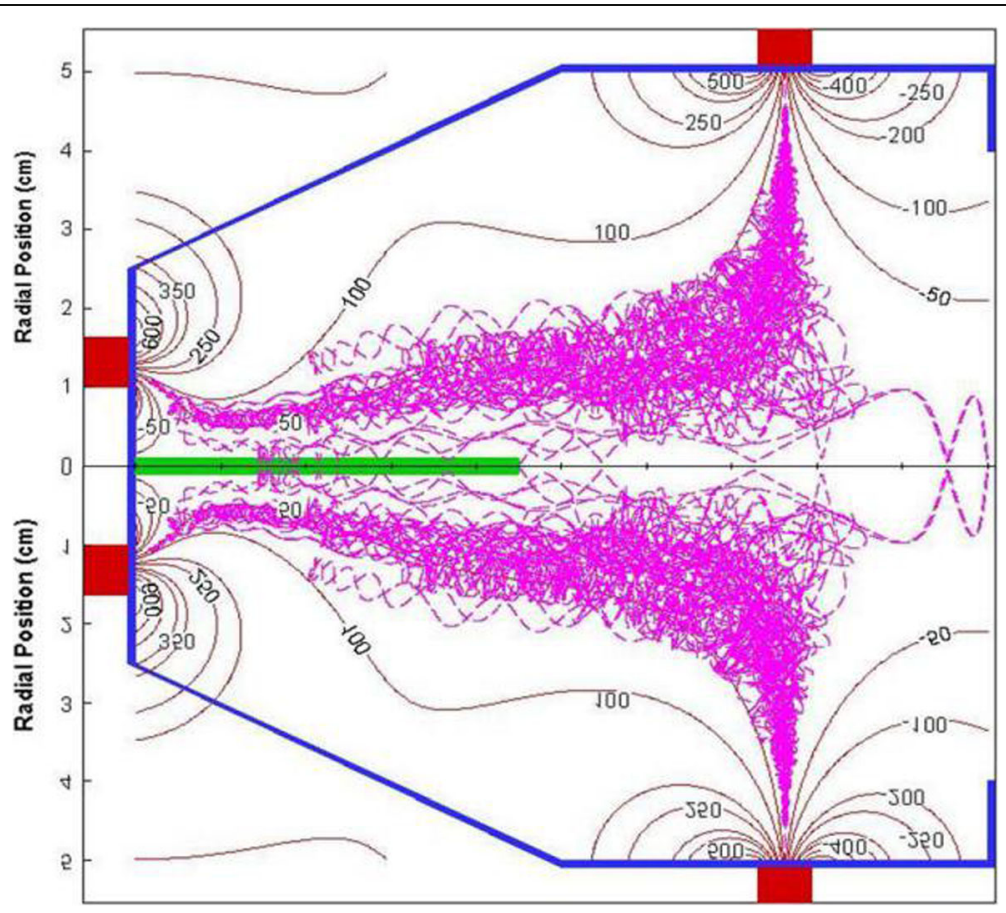

Fig. 10 Charge particle trajectory in an Hall effect ion thruster
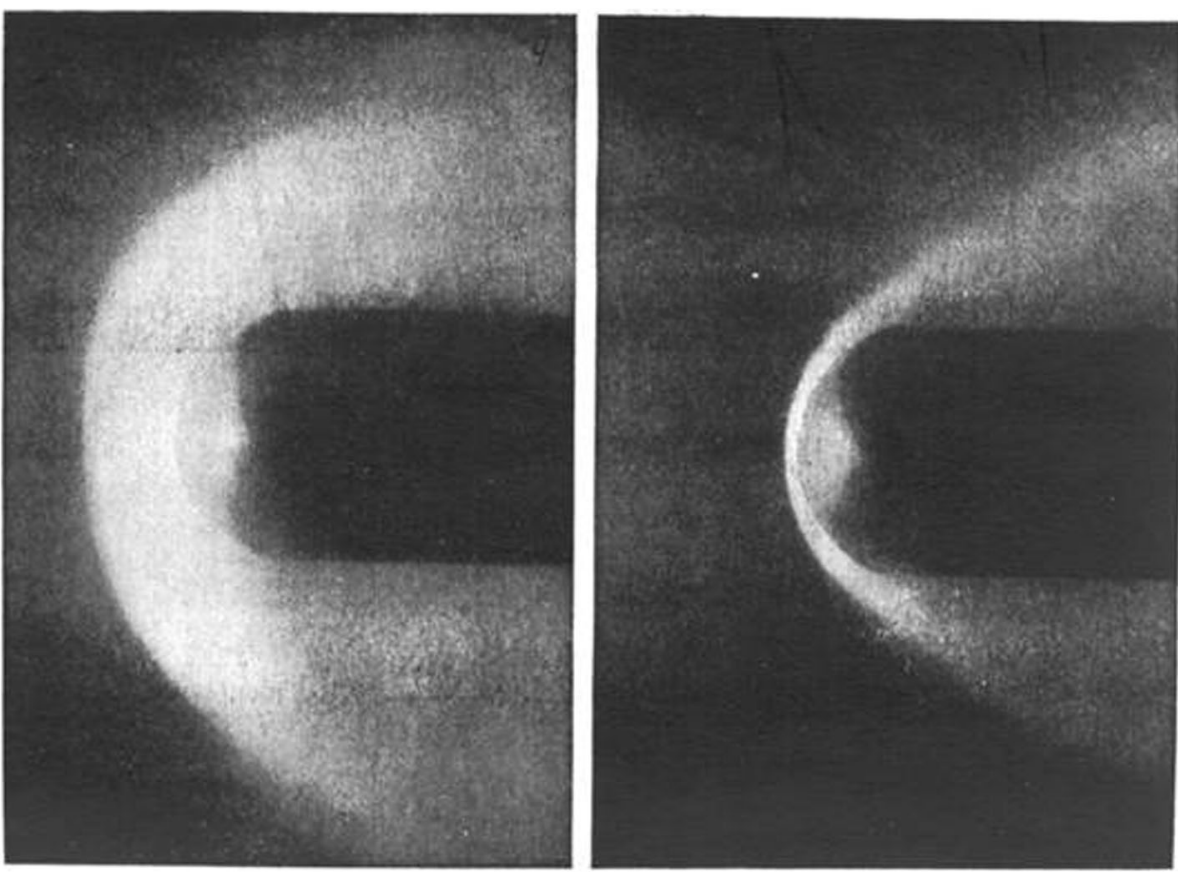

Fig. 11 Magnetic field effect on bow shock standoff distance 
cross section reduction, antenna and integrated circuit design. The system of linear, partial differential equations can be presented as [34, 39];

$$
\begin{aligned}
& \partial B / \partial t+\nabla \times E=0 \\
& \partial D / \partial t-\nabla \times h+J=0 \\
& \nabla \cdot D=\rho_{e} \\
& \nabla \cdot B=0
\end{aligned}
$$

It is worth noting that all these laws follow a single physical concept, and an understanding of its rationality is invaluable in order to apply these equations. The Faraday's induction law simply states that a changing magnetic flux density in time will induce electric field intensity in path surrounding it. The generalized Ampere's law on a varying-time frame defines the displacement electric current and the relationship between the magnetic intensity and the conductive electric current. The Gauss's law for electric displacement $D$ states that an electric field must be originated and terminated on electric charges and it is a definition of the electric charge density. In contrast, the Gauss's law for magnetic flux $B$ does not permit a source in an electromagnetic field; the lines of magnetic flux have no beginning or end in an electromagnetic field like the vorticity of inviscid fluid dynamics. The electromagnetic field equations consist of six dependent variables: the electric field intensity $E$, the magnetic field intensity $H$, the electric flux density $D$, the magnetic flux density $B$, electric current density $J$, and the electric charge density $\rho_{e}$. The system of equations is not necessarily linear independent under certain circumstance.

From a pure mathematical point of view, the Maxwell's equations have a closure issue, namely, the number of dependent variables is unbalanced by the number of unknowns. Meanwhile for problem solving, the material media property also needs to achieve the physical fidelity. This dilemma is removed by the constitutive relations. The most general form is based on the relativistic consideration to be described by four tensors, in a four dimensional spaces, and they are Lorentz-covariants [39]. In an isotropic media, the required constitutive properties are simply scalar quantities;

$$
\begin{aligned}
& D=\varepsilon E \\
& B=\mu H
\end{aligned}
$$

For solving the Maxwell equation in the time domain, which constitutes a hyperbolic partial differential system, the initial values and boundary conditions for all dependent variables are required. The boundary conditions, especially the compatible conditions across media interfaces and at the farfield are paramount for accurate electromagnetic computational simulations, which must be consistent with the appropriate media interface and the no-reflection farfield boundary conditions [19]. On a stationary frame of reference, the velocity of the moving control surface vanishes, and the boundary condition acquires a simpler form. It is convenient to describe the boundary condition by the tangential and normal components on the media interface [39].

$$
\begin{aligned}
& n \times\left(E_{1}-E_{2}\right)=0, \quad n \cdot\left(D_{1}-D_{2}\right)=\rho_{s} \\
& n \cdot\left(B_{1}-B_{2}\right)=0, \quad n \times\left(H_{1}-H_{2}\right)=J_{s}
\end{aligned}
$$

The first two boundary conditions state that the tangential electric field intensity $E$ and the normal component of the magnetic flux density $B$ are continuous across the interface boundary. Conversely, the difference in normal component of the electric 
displacement $D$ must be balanced by the surface charge density. Finally, the discontinuity of the tangential components of the magnetic field strength $H$ is determined by the surface current density $J_{s}$. These boundary conditions are paramount in analyzing the electromagnetic phenomena.

Alfven first initiated the field of magnetohydrodynamics (MHD) by integrating the electromagnetics with aerodynamics, which was originally derived for investigating the astrophysics and geophysics phenomena [33]. The basic approximation treats plasma as a multi-fluid medium consisting of electrons, positively charged ions, and neutral particles on the framework of wave dynamics. The displacement electric current of plasma is neglected in comparison with respect to the conductive components. Thus the scalar electric conductivity is determined by the generalized Ohm's law, which is consistent with the basic premise of the complete formulation [20]. As a consequence, MHD formulation is only valid for studying ionized gas dynamics with a frequency range limited to microwave spectrum. For the same reason, the explicitly imposed approximation on plasma composition has led to some serious limitations on accurate analyses for the detailed plasma dynamics behavior in hypersonic flows. Indeed, the simplified approach fails to yield a predictive accuracy for feasibility studies of the scramjet MHD bypass innovation of the AJAX vehicle concept [29]. It is a glaring example by applying a classic theory beyond its intended scope. Regardless, the simplified analytic formulation of MHD permits a thorough investigation of the plasma dynamics in an electromagnetic field and reveals the most unique and intrinsic characteristics of air plasma.

\section{Radiation in hypersonic flows}

The radiative energy transfer coincides with quantum transitions which occur at a highly excited energy states of atoms or molecules. It is an intrinsic physical phenomenon of the high enthalpy gas in hypersonic flows. The energy transfer at quantum transitions has been divided into three groups according to the continuity criterion of its energy spectrum, and by the initial and final states of the transition as the bound-bound, bound-free, and free-free types. The bound-bound transitions take place in atoms and molecules quantum transition from one discrete state to another. These transitions follow the permissible quantum jumps and the energy transfer is limited to an unit of $h v$. The cross sections of absorption are associated with the photon mean free path, which at the standard atmospheric condition is around $10^{-10} \mathrm{~cm}$ [30].

The bound-free transition is resulting from the energy transfer that exceeds the bounding energy of electrons to nuclei; the electrons become free. The excessive energy is transformed into the kinetic energy of free electrons. Thus, the energy transfer can have continuous spectra. In the case of a reverse transition, the bonded electron will release the energy by photons. The free-free transition is also known as the Bremsstrahlung (brake down), because the motion of free electrons is slowing down in the field of the ion, and loses a part of its energy by the radiation process. Its emission and absorption has a continuous spectrum $[29,30]$.

The radiative field in time and space must be described by the distribution function of its intensity with respect to its frequency $v$ and direction of the energy transfer $\Omega$. The radiation frequency spectrum covers a wide range from radio wave, infrared, visible, ultraviolet, $\mathrm{x}$-ray to gamma-ray, $10^{3}$ to $10^{22} \mathrm{~Hz}$, by wave lengths it varies from 
$10^{-3}$ to $10^{-12} \mathrm{~m}$. The radiative energy is defined by the spectral radiation intensity per unit time and area;

$$
I_{v}(r, \Omega, t)=h v f(v, r, \Omega, t)
$$

The portion of spectra associated with hypersonic flows for thermal energy transfer however is mostly concentrated in the infrared, visible, and ultraviolet spectra. For a visible radiation, the characteristic medium temperatures are in the order from 7000 to $13,000 \mathrm{k}$. The spectrum range of thermal radiation in frequency and wavelength, as well as, in term of electromagnetic wave types is presented in Fig. 12. The radiative energy transfer in hypersonic flow is rather limited in a narrow frequency range from $4.0 \times 10^{5}$ to $7.9 \times 10^{5} \mathrm{GHz}[29]$.

A radiation field is described by the absorption, emission, and scattering of radiative intensities by its frequency, position, and the direction of energy transfer. The integral result over the solid angle is a vector quantity known as the spectral radiant energy density or the radiating energy flux within a solid angle $\Omega$,

$$
U_{\nu}(s, t)=h v \int_{4 \pi} f(v, s, \Omega, t) d \Omega=\int_{4 \pi} I_{\nu}(s, \Omega, t) d \Omega
$$

The classic theories of radiative energy transfer are derived from the equilibrium radiative condition [40-42]. If the radiating field is isotropic, the radiation can be described as a universal function of the frequency and temperature. From the statistics quantum mechanics, the classic spectral energy density has been derived by Planck in 1914, and it is the amount of radiated energy per unit volume at the equilibrium state at a frequency $v$.

$$
U_{v}=\left(8 \pi h v^{3} / c^{3}\right) /\left(e^{h v / k T}-1\right)
$$

The spectral intensity in the isotropic radiation condition is given by the Planck distribution function or simply the Planck's function.

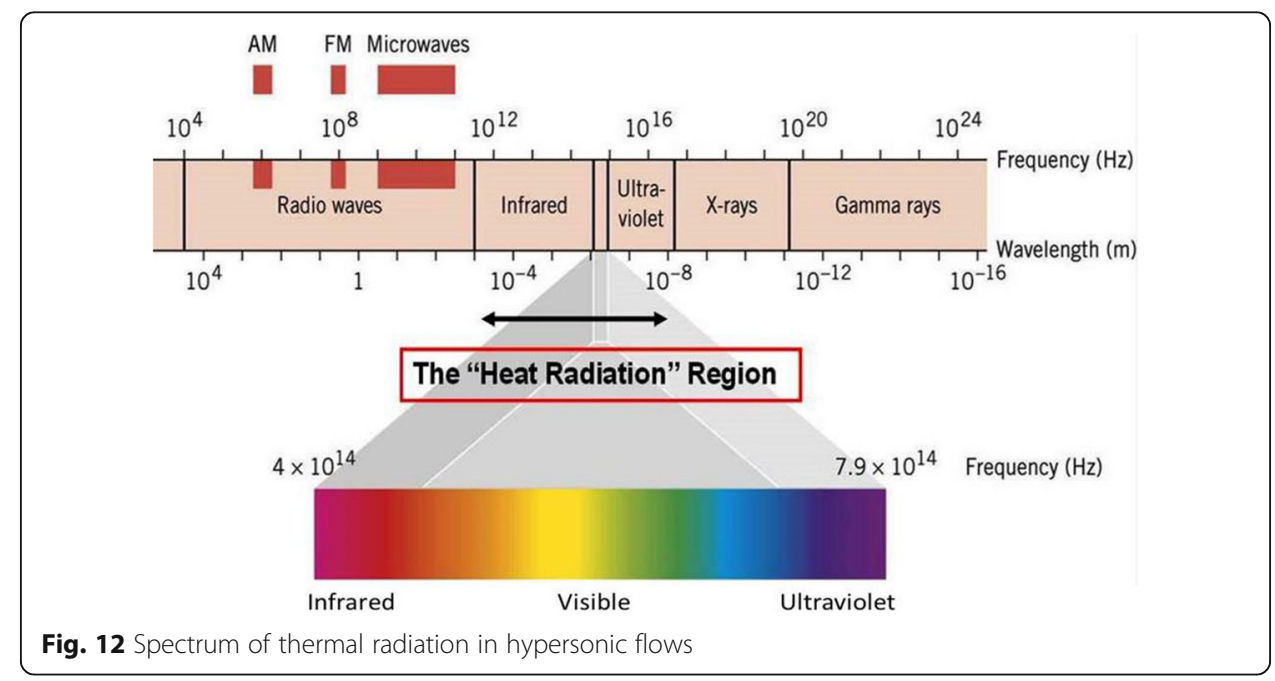




$$
I_{v}=\left(2 h v^{3} / c^{2}\right) /\left(e^{h v / k T}-1\right)
$$

The radiation intensity given by the Planck function is also called the black body radiation, emitted by a perfect radiator or the black body [40,42]. The spectral energy density from a black body radiation is provided by the Stefan's law, by integrating the Planck function over all frequency spectra to get the coefficient $\sigma=\left(8 \pi^{5} \kappa^{4} / 15 c^{3} h^{3}\right)$ which is the well-known Stefan-Boltzmann constant [42]. In the cgs system, it has the value of $5.67 \times 10^{-5} \mathrm{erg} /\left(\mathrm{cm}^{2} \cdot \mathrm{sec} \cdot k^{4}\right)$. The amount of radiant energy is given by the value per unit time, and area over all frequencies is therefore the well-known result $\sigma T^{4}$.

In most hypersonic flights in ionized air with a temperature around $10,000 \mathrm{k}$ or higher, and an electron number density of $10^{13} / \mathrm{cm}^{3}$, the radiation is not in equilibrium state but the radiative pressure is negligible [42]. The radiative energy transfer manifests itself by exchanging with the surrounding gas medium or the free space that can be quite appreciable. The mechanism of radiation is remarkably different from the conductive and convective heating by virtue of its light-speed wave propagation in comparison with the sonic collision process. Therefore, the radiation heat transfer is generally considered to be instantaneous and independent of time in comparison with gas motion. The radiant energy and the flux of energy are resembled. For this reason, an isotropic flux can be given by the divergence of the radiation flux.

The total energy transfer per unit volume and time is found by integrating the difference between spontaneous emission and absorption over the retire solid angle and spectral frequencies; $Q=\int_{0}^{\infty} d v \int k_{v}\left(I_{v p}-I_{v}\right) d \Omega$. Since the total energy transfer is nearly independent from the time scale in gas motion. The total energy transfer in hypersonic flow is equal to the divergence of the integrated radiation flux;

$$
\begin{aligned}
& Q=\int_{0}^{\infty} \nabla \cdot q d v=\nabla \cdot q \\
& q=\int \cos \theta I_{v}(\Omega) d \Omega
\end{aligned}
$$

where $\theta$ is the angle of direction cosine between the radiation propagation and the outward normal of the control surface.

A breakdown of the total stagnation-point heat transfer distributions on the Stardust probe in earth reentry is presented in Fig. 13, together with an illustration of the solid angle for radiation, as well as, the surface shear stress pattern and temperature contour over the heat shield. At the peak heating load condition, the probe is traveling at a speed of $11.14 \mathrm{~km} / \mathrm{s}$, the flight condition is characterized by the ambient temperature of $238.5 \mathrm{~K}$ and the air density of $2.34 \times 10^{-7} \mathrm{~g} / \mathrm{cm}^{3}$. The maximum numerically simulated heat load on the capsule is $1.19 \times 10^{3} \mathrm{~W} / \mathrm{cm}^{2}$ at the stagnation point. The total heat transfer rate does not include the active ablation, and is in a very good agreement with the results by Olynick et al. of $1.2 \times 10^{3} \mathrm{~W} / \mathrm{cm}^{2}$ [22] and agrees equally well with the laminar flow result by Park at $1.189 \times 10^{3} / \mathrm{cm}^{2}$ [23]. The radiative heat transfer rate at the stagnation point yields a value of $248 \mathrm{w} / \mathrm{cm}^{2}$, which is also comparable with other investigations. From the distributions of heat transfer rates over the capsule frontal 


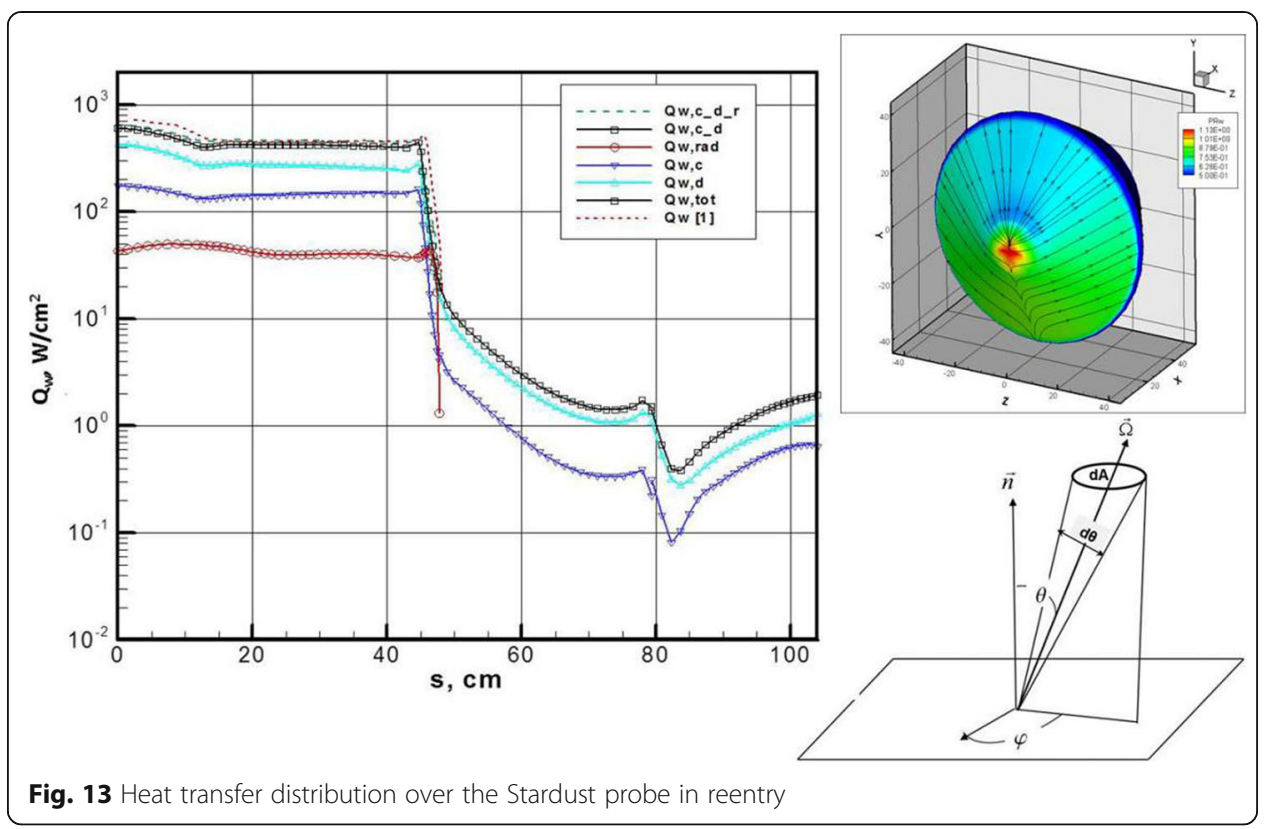

surface, the increasing contribution in hypersonic flows from the diffusive (convective) $\mathrm{Q}_{\mathrm{w}, \mathrm{d}}$ and radiative heat transfer $\mathrm{Q}_{\mathrm{w}, \mathrm{r}}$ over the conductive $\mathrm{Q}_{\mathrm{w}, \mathrm{c}}$ heat transfer becomes clear. Especially, the radiative heat transfer in a hypersonic interplanetary reentry can reach an amount as high as $35 \%$ of the total heat transfer [42].

Traditionally, radiation has been described as a particle phenomenon for energy transmission by photons or light quanta, but on the frame work of an electromagnetic field theory, radiation is described by the wave dynamics not only by its energy content but also by its optical behavior. Therefore, the basic radiative mechanisms are given by the emission and absorption of electromagnetic waves during the quantum jumps. The amount of transferred energy is actually the difference between two quantum states known as the selective absorption. Along the beam path, the propagating radiation interacts with the electromagnetic field and appears as the scattering of waves.

According to the kinetic theory, all the radiating intensities are uniquely related to the photon distribution function $f(v, s, \Omega, t)$. The radiant intensity is an integrable quantity in a spectral interval and in a range of solid angle $d \Omega$ along the direction of unit vector $\Omega$ as it has been defined by Eq. (27). There are really five detailed basic mechanisms in the radiation heat transfer process: the spectral absorption, emission, scattering, reflection from media interface, and re-absorption. The spectral emission is more complex, traditionally it has been categorized as spontaneous, induced or medium emissivity, because it depends on the state and properties of the emitting medium. The spectral radiation scattering coefficient $\sigma_{v}(s)$ gives the scattering rate per unit volume for radiation propagated in an elemental solid angle $d \Omega$, therefore the scattering of radiative energy is as follows; $\sigma_{\nu}(s) I_{\nu}(s, \Omega, t) d \Omega$.

Attenuation of radiative energy in a medium can be incurred by absorption, reflection, and scattering, and the opacity of a medium is a measurement for the attenuation of the transmitted radiation energy. By definition, the spectral scattering indicatrix $p(s$, $\left.\Omega^{\prime}, \Omega, v^{\prime}, v\right)$ forming the radiative wave scattering is a probability function for scattering 
to the direction from $\Omega$ to direction $\Omega^{\prime}$ and to the frequency $v^{\prime}$ from another frequency $v$. The rate of energy transfer between two states will have a value of $(1 / 4 \pi) \sigma_{v}(s) I_{\nu}(s$, $\left.\Omega^{\prime}, t\right) p\left(s, \Omega^{\prime}, \Omega, v^{\prime}, v\right) d \Omega^{\prime} d v^{\prime}$.

In summary, the radiating mechanisms are generated by the kinetics of photons or light quanta which are treatable by the integro-differential Boltzmann equation for the photon distribution function in phase space. The radiation rate equation is $[29,42]$;

$$
\frac{1}{c} \frac{\partial I_{\nu}(s, \Omega, t)}{\partial t}+\frac{\partial I_{\nu}(s, \Omega, t)}{\partial s}+\left[k_{v}(s)+\sigma_{\nu}(s)\right] I_{\nu}(s, \Omega, t)=I_{\nu}^{e m}(s, t)+\frac{\sigma_{\nu}(s)}{4 \pi} \int_{v=0}^{\infty} \int_{\Omega=4 \pi} p\left(s, \Omega^{\prime}, \Omega, v^{\prime}, v\right) I_{\nu^{\prime}}\left(s, \Omega^{\prime}, t\right) d \Omega^{\prime} d v^{\prime}
$$

The two leading terms on the left-hand-side of the equation are the total derivative of the radiative intensity, and the convective velocity is the speed of light along the direction of the solid angle. The third term represents the spectral intensity associated with the absorption and scattering with the coefficient of $k_{v}(s)$ and $\sigma_{v}(s)$ respectively. The first term on the right-hand-side of the equation represents the amount of spectral emission in the medium, and the last term describes the contribution to the radiative rate of change by the radiative wave scattering.

The time-dependent term of the time radiative rate equation is scaled by the speed of light. It is intuitively obvious that the derivative with respect to time is therefore negligible in comparison with the spatial derivative. Thus the time-independent radiative rate equation reduces to the following form;

$$
\partial I_{v}(s, \Omega) / \partial s+\left[k_{v}(s)+\sigma_{v}(s)\right] I_{v}(s, \Omega)=I_{v}^{e m}(s)+\frac{\sigma_{v}(s)}{4 \pi} \int_{v=0}^{\infty} \int_{\Omega=4 \pi} p\left(s, \Omega^{\prime}, \Omega, v^{\prime}, v\right) I_{v^{\prime}}\left(s, \Omega^{\prime}\right) d \Omega^{\prime} d v^{\prime}
$$

An outstanding characteristic of the propagating radiating ray in a gas medium is its opaqueness, which is caused by the amplitude attenuation of the electromagnetic waves by collision and non-collision processes. The latter is incurred by scattering of photons by diffraction or by penetration of photon into particles and by change of direction from refraction. As a consequence, the medium of radiation propagation has been classified as the optical thick or the optical thin. However, in most hypersonic flows, the effect of the radiative wave scattering phenomenon is often negligible in comparison with absorption and emission.

One of the most challenging research areas of radiation is the evaluation of the emission, absorption, scattering coefficients and the spectral scattering indicatrix. These coefficients are optical property of a gas medium, which depends exclusively on the thermodynamic state of the transmitting gaseous medium, and must be derived from a combination of the quantum mechanics and quantum optical physics. As an example, the typical air spectral absorption coefficient distributions are displayed in Fig. 14, at two different temperatures of $5000 \mathrm{k}$ and $20,000 \mathrm{k}$, and one atmosphere pressure. It is clearly shown that the magnitudes and distributions of absorption spectral coefficient as a function of wavenumber are entirely different from each other, which reflects the different excited internal energy states of the air mixture [42].

The radiation rate equation, Eq. (33), is hyperbolic partial differential equation like that of the Euler equations; its solution has a domain dependent property. Therefore, the Milne-Eddington approximation is applicable [41]. The approximation considers 

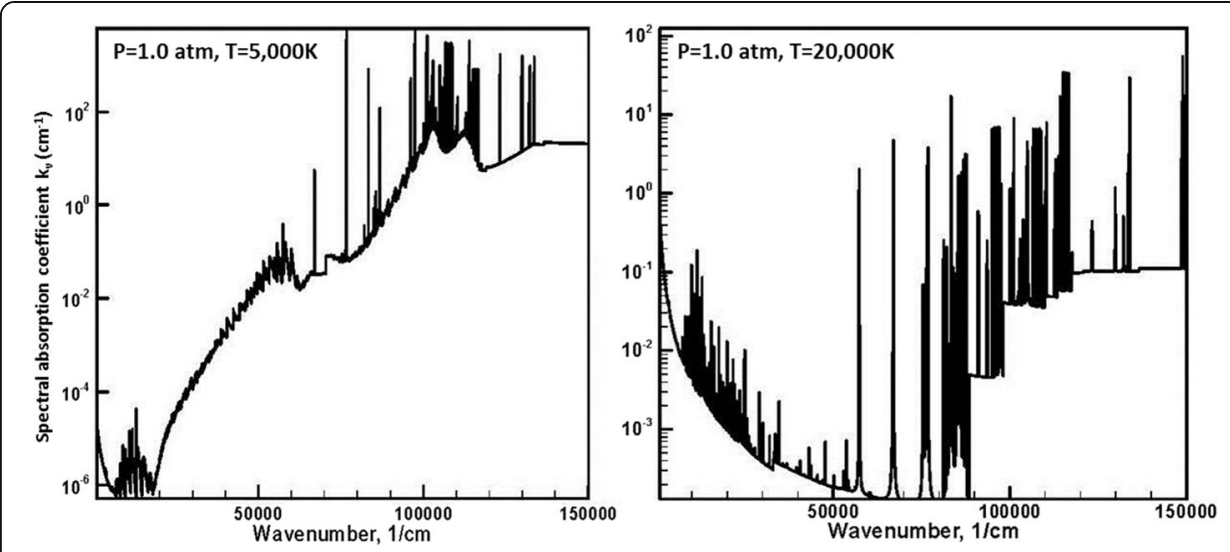

Fig. 14 Spectral absorption coefficients in different air mixture temperatures

the radiation is transmitting in one direction, and all directional components have a value independent of the solid angle thus the local radiation in each coordinate direction is isotropic. The analytical and computational methods adopting this approximation are the half-moment method, spherical harmonic method, and discrete ordinate method [41, 42]. A unique feature of the discrete ordinate method stands out by a coordinate transformation. The basic idea is transforming the coordinate of the radiation rate equation for a gray medium from the Cartesian frame onto coordinates of the propagating radiative wave by the chain rule of differentiation. The orientation of the radiation intensity is further discretized into many directions in the transformed coordinates into a set of simultaneous partial differential equations [42, 43].

In practical applications, the ray tracing technique is often adopted by recent numerical simulations for radiation heat transfer. This technique is based on optical physics and the procedures of geometric optics through which are applied to the radiative exchange process. The radiative energy flux is actually evaluating on each single radiating ray by integration $[25,29,42]$. The spectral group approximation is another numerical approximation to solve the radiation rate equation, Eq. (33). The numerical procedure is dividing the complete spectrum domain into subgroups according to their frequency and solving them individually as an isotropic medium first, and summing all separated solutions afterwards. At least a few hundred spectral subgroups are required by a multi-spectral group approximation to provide an adequate representation of the complete radiating spectrum.

The spectral line-by-line integration and the Monte Carlo method become the cutting-edge approaches for radiation energy transfer [29, 42]. The Monte-Carlo methods have been used to simulate directly the photon dynamics. In solving the radiation rate equation, the Monte-Carlo algorithm can accelerate the convergence through coupling the forward and adjoint radiation transport equations [44];

$$
\Omega \cdot \nabla I(s, \Omega)+k_{v} I(s, \Omega)=\sigma_{v}(s) \int_{\Omega} I\left(s, \Omega^{\prime}\right) p\left(s, \Omega, \Omega^{\prime}\right) d \Omega^{\prime}+I_{v}^{e m}(s, \Omega)
$$

The solution of the radiation intensity is expressed in terms of a volume Green's function, which leads to an adjoint integro-differential equation as; 


$$
-\Omega \cdot \nabla I^{\prime}(s, \Omega)+k_{v} I^{\prime}(s, \Omega)=\sigma_{v}(s) \int_{\Omega} I^{\prime}\left(s, \Omega^{\prime}\right) p\left(s, \Omega, \Omega^{\prime}\right) d \Omega^{\prime}+I_{v}^{\prime e m}(s, \Omega)
$$

All basic research processes for solving the radiation transport equation using Monte Carlo methods require the support by the high-performance parallel computing technology. The solving procedure is akin to the gas kinetic theory, but is directly simulating the photons dynamics based on the integro-differential Boltzmann equation to stay on the leading edge of aerodynamic science $[29,42]$.

There are only a limited number of high-enthalpy ground-based experimental facilities that are focusing on the optical properties of radiating gas [45]. Thus the validations for the numerical modelling and simulation are relying solely on the flight test data. As a consequence, only a global assessment or engineering calibration for the physical fidelity of radiating energy transfer is achievable at the present.

A summary of the state-of-art progress on radiation energy transfer in high enthalpy hypersonic flows is presented by Fig. 15, which exhibits the computational capability and its calibrations with flight data [29]. The FIRE II (Flight Investigation of Reentry Environment) space vehicle is constructed by a circular arc forebody and a conical afterbody, and reenters earth at a nominal velocity of $11.35 \mathrm{~km} / \mathrm{s}$. The thermal shield covering the fore face consists of a layer of phenolic-asbestos composite material. The flight data was collected by thermocouples and forward-looking radiometers between the initial heat pulse and the peak heating load condition at the altitude range from 77 to $37 \mathrm{~km}$. The significant discrepancy between computational simulations and the limited data reflects the need for a continuing improvement to the radiation research of the hypersonic flows.

\section{Hypersonic interdisciplinary governing equations}

The physically realistic hypersonic flow in the continuum regime has outgrown the reach of the classic gas kinetic theory. From the basic approach of adopting the binary elastic collision, the internal degrees of freedom of gas particles in microscopic scale are not taken into consideration. In order to remedy the deficiency, the collision model needs to describe the interactions between particles by inelastic collisions. Meanwhile, the quantum phenomena impose formidable challenges both in concepts and basic understandings that are prohibitive for any rudimentary and systematic analytic development. For now, investigations are conducted mainly by interdisciplinary investigations with physics-based models derived from all the pertaining scientific disciplines integrating into aerodynamics.

The frame work of the most recent hypersonic flow research is built on accumulated multidisciplinary knowledge from the high-speed propulsion with chemical kinetics, and from the magnetohydrodynamics with electromagnetics. Nearly all hypersonic flows are immersed in a high enthalpy but weakly ionized air by shock wave compression. Although the electromagnetic effects are drastically altering the characteristics of flow medium, the magnitudes of all the remote-acting forces still remain at a perturbation level in comparison to aerodynamic inertia. The relative magnitude between aerodynamic and electromagnetic forces is measured by the magnetic Reynolds number, which is defined as $R_{m}=u L /(\sigma \mu)^{-1}=\sigma \mu u_{\infty} L[19,20,35]$. The value of this demarcation similitude is less than unity in most hypersonic flows, only in an MHD thruster its 


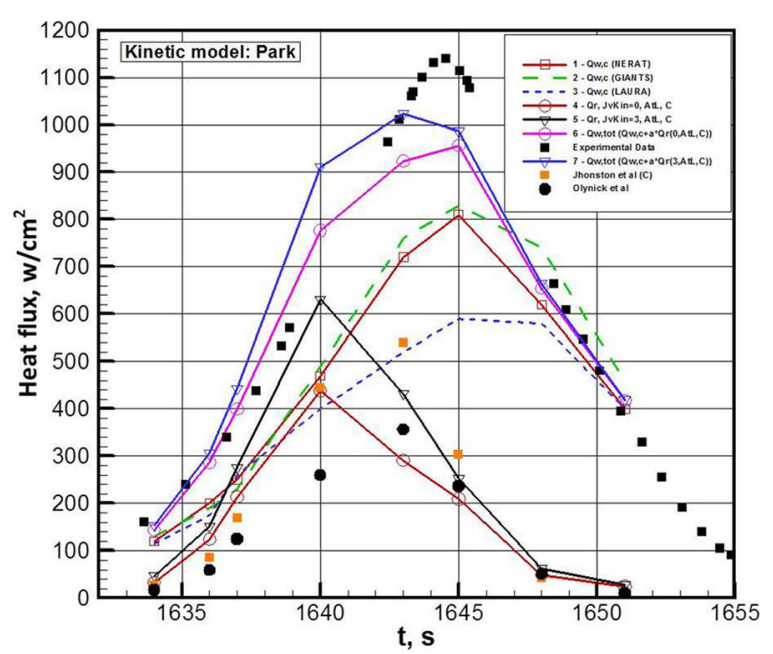

Fig. 15 Verification of numerical simulation with FIRE-II flight data

value reaches unity, and attains a value around one hundred in the controlled thermonuclear reaction [20].

The low magnetic Reynolds number approximation is fully justified for most hypersonic flows of aerodynamic interest. Based on the stipulated condition, the Faraday's induction law can be decoupled from the interdisciplinary governing equations [19, 33]. The momentum exchange within the control volume is now balanced by the electrostatic force $\rho_{e} E$ and Lorentz acceleration $J \times B$, which are appended to the right-handside of the momentum conservation equation. The conservation of energy of the complete system needs to include the energy cascading and transmitting by quantum jumps from the translational mode to vibrational $Q_{t, v}$ as well as the electronic $Q_{t, e}$ modes. The Joule heating, $E \cdot J$ of the ionized air and the radiative heat transfers are also added to the conservation energy equation as the source terms. The effects of transport properties are reinforced by the gas kinetic theory, which is always the key coupling mechanism between conservation mass, momentum, and global energy equations.

The quantum jumps of the internal degrees of freedom between translational, vibrational, and electronic excitations are included to the conservation energy laws for each individual species. The identical species with different internal degrees of freedom are also identified as different chemical compositions; thus the vibrational energy conservation equations for polyatomic molecular species are included in the governing equations together with the energy conservation equation of the electronic excitation. The complete interdisciplinary governing equations acquire the following forms [19, 29];

$$
\begin{aligned}
& \partial \rho_{i} / \partial t+\nabla \cdot\left[\rho_{i}\left(u+u_{i}\right)\right]=d w_{i} / d t \\
& \partial \rho u / \partial t+\nabla \cdot(\rho u u+p-\tau)=\rho_{e} E+(J \times B) \\
& \left.\partial \rho_{i} e_{v i} / \partial t+\nabla \cdot\left[\rho_{i}\left(u+u_{i}\right) e_{v i}+q_{v i}\right)\right]=\left(d w_{v i} / d t\right) e_{v i}+Q_{v, \Sigma} \\
& \left.\partial \rho_{i} e_{e} / \partial t+\nabla \cdot\left[\rho_{i}\left(u+u_{i}\right) e_{e}+u \cdot p_{e}+q_{e}\right)\right]=\left(d w_{e} / d t\right) e_{e}+E \cdot J \\
& +\left[\rho_{e} E+(J \times B)\right] \cdot\left(u+u_{i}\right)+Q_{e, \Sigma}
\end{aligned}
$$




$$
\partial \rho e / \partial t+\nabla \cdot\left[\rho e u-\kappa \nabla T+\sum \rho_{i} u_{i} h_{i}+q_{r a d}+u \cdot p+u \cdot \tau\right]+Q_{t, v}-Q_{t, e}=E \cdot J
$$

In Eqs. (36), (38), and (39), the production and depletion rates of each chemical species, and the same species but of different internal mods have been included. The detailed rate of change is described by Eq. (7). The specific internal energy of the air mixture including the electronic energy now becomes;

$$
\rho e=\sum_{i \neq e} \rho_{i}\left(e_{i}+u \cdot u / 2\right)+\sum_{i \neq e} \rho_{i} \Delta H_{i}^{o}+\sum_{i \neq e} \rho_{i} e_{v i}+\sum \rho_{e}\left(e_{e}+u_{e} \cdot u_{e} / 2\right)
$$

The quantum transitions and energy redistribution among internal excitations $Q_{t}, v$, $Q_{v, v}, Q_{t, e}$ are modeled including the relaxation phenomenon. The notations $Q_{v, \Sigma}$ and $Q_{e, \Sigma}$ in Eq. (38) and (39) represent all permissible quantum transitions for vibrational and electronic modes respectively. These models do not make any distinction between either by a single quantum number jump (ladder climbing theory) or the multiply quanta jump (big bang theory), but only evaluate the energy cascading among different species and quantum states.

The relaxation time scale between translational and vibrational mode by Landau et al. [46] is described by the principle of detailed balance;

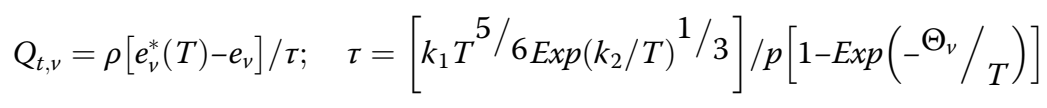

The relaxation time scales among vibration-vibration quantum jumps are approximated by an outstanding correlation from experimental observations due to Millikan et al. [47] from a wide range of experimental data and by different experimental techniques.

$$
Q_{v, v}=\rho\left[e_{v}^{*}(T)-e_{v}\right] / \tau ; \quad \tau=1.16 \times 10^{-3} M_{12}^{1 / 2 \Theta_{v}^{4} / 3}\left[\left(T^{-1 / 3}-0.15 M_{12}-18.42\right]\right.
$$

where the $M_{12}$ designates the reduced mass of the interacting species, $M_{12}=M_{1} M_{2} /$ $\left(M_{1}+M_{2}\right)$.

On the other hand, the quantum transition between electron-ion collisions is a pure empirical model to appear as [48];

$$
\left.\left.Q_{e, \mathrm{~V}}=2 \times 10^{-16} \sum_{\nu} n_{e} n_{i} \omega_{e, v} P_{1,0, v}\left\{e^{-\left(1.44 \omega_{e, v} / T e\right)} /\left[1-e^{-\left(1.44 \omega_{e, v} / T e\right)}\right]-e^{-\left(1.44 \omega_{e, v} / T v\right.}\right) /\left[1+e^{-\left(1.44 \omega_{e, v} / T v\right.}\right)\right]\right\}
$$

where the empirical determined coefficient is $P_{1,0, V}=0.45 \times 10^{-10} \exp -\left(10000 / T_{e}\right)$.

There are numerous quantum jump models being applied from the dissociation biased vibration-vibration quantum transitions, vibration-electronic transition, electron-ion transition to the neural-electron collision [19, 23, 29]. Some of these models are developed from a rigorous theoretic consideration but others are simply devised by empirical means. Therefore, there are ample rooms for improvement as a promising new frontier for basic research by either experimental or the ab initio (from the beginning) approaches [18].

The high-enthalpy transport properties in a heterogeneous, nonequilibrium chemically reacting air mixture play a dominant role for momentum and energy transfer. The molecular viscosity, diffusion, and thermal conductivity originated from the 
nonequilibrium collision process, are the landmark achievements from the gas kinetic theory by describing it with an inter-molecular potential function [2, 20, 49]. These classic analytic results for collision cross sections and collision integrations are still valid but must be generated by different inter-molecular potentials according to the gas thermodynamic states $[28,29,50]$.

The transport coefficients of a single molecular species are obtained from the Maxwell-Boltzmann distribution by the Chapman-Enskog expansion [2, 20]. The molecular viscosity coefficient derived from the gas kinetic theory with the collision cross sections and collision integrals is,

$$
\mu=2.67 \times 10^{-5} \sqrt{M_{i} T} / \sigma_{i}^{2} \Omega^{(2,2)}
$$

The molecular diffusion coefficients in most hypersonic flows are focused only on the ordinary diffusion generated by the gradient of species concentration. The coupled Dufour and Soret effects are omitted, which are consequences between the high pressure and temperature gradients on the viscosity. These detailed descriptions have been fully articulated by the Onsager reciprocal theorem [19, 29]. Again, by neglecting the ambipolar diffusion from the ionized air, only the binary diffusion coefficient is necessary for the high-enthalpy air,

$$
d_{i, j}=1.858 \times 10^{-3} \sqrt{T^{3}\left(M_{i}+M_{j}\right) / M_{i} M_{j}} / \sigma_{i, j}^{2} \Omega^{(1,1)}
$$

The classic molecular thermal conduction coefficients for the monatomic and polyatomic molecules are known;

$$
\begin{aligned}
& \kappa_{i, m}=1.989 \times 10^{-4} \sqrt{T / M_{i}} / \sigma_{i}^{2} \Omega^{(2,2)} \\
& \kappa_{i, p}=2.519 \times 10^{-4} \sqrt{T / M_{i}} \in / \sigma_{i}^{2} \Omega^{(2,2)}
\end{aligned}
$$

The intermolecular potentials vary from one gas species to another and according to their respective thermodynamic states, but the collision cross section of air mixture generally lies in between the range from 2 to 4 Angstroms $\left(10^{-8} \mathrm{~cm}\right)$. The typical volume of a gas molecule is approximately around $10^{-23}$ per cubic centimeter at the standard atmospheric condition. Therefore, the intermolecular forces are really the short range forces acting on colliding particles. The most widely adopted molecular potential is the classic Lennard-Jones 6-12 potential [2],

$$
\phi(r)=4 \phi_{o}\left[(\sigma / r)^{12}-(\sigma / r)^{6}\right]
$$

This potential function has been universally applied for gas species up to a temperature of $2000 \mathrm{k}$. For a gas in a bounded state or only when the vibrational excitation is dominated, the Morse potential has been used [19, 48, 50],

$$
\phi(r)=\phi_{e q}\left\{e^{\left[-2(c / \sigma)\left(r-r_{e q}\right)\right]}-2 e^{\left[-2(c / \sigma)\left(r-r_{e q}\right)\right]}\right\} ; \quad \phi_{e q}=\phi\left(r_{e q}\right)
$$

In the dissociation temperature range, an exponential repulsive model is often adopted.

$$
\phi(r)=\phi_{o} e^{-r / \rho}
$$


For the ionized gas, the collisions mainly involve the ion-ion and electron-electron collision, and the screened Coulomb potential is utilized for the collision integration calculations [50];

$$
\phi(r)=\left(e^{2} / r\right) e^{-r / d} ; d=\left(\varepsilon \kappa T / e^{2} n\right)
$$

The required collision integrals and cross sections for the high enthalpy hypersonic flow have been obtained by the Lenard-Jones potential for non-polarized molecules, and by a polarizability model for ion-neutral non-resonant collisions [50]. The transport properties generated from the gas kinetic theory for individual molecular species are used to determine the global property for a gas mixture by the Wilke's mixing rule $[29,49]$.

The viscosity and thermal conductivity coefficients of ionized hypersonic flows are depicted by Figs. 16 and 17 respectively. Figure 16 presents the high-temperature air viscosity coefficient distributions covering a limited temperature range up to $20,000 \mathrm{k}$, and includes some bench-mark results by other formulations for the purpose of comparison. An inflection point of the viscosity coefficient in temperature appears at a value beyond 10,000 k, and decreases as the air temperature increases further. From the shared knowledge, the viscosity of the ionized air will gradually increase again beyond the displayed temperature range [51].

Figure 17 depicts the thermal conductivity coefficients of high temperature air plasma spanning the temperature range up to $30,000 \mathrm{k}$ [52]. All the presented numerical results include a wide variation of physics-based models and exhibit a considerable amount of disparities from each other. Although there is substantial progress in the kinetic theory of gases for evaluating transport properties by the rational intermolecular potentials, yet the calculated transport coefficients still yield an unacceptable difference of $16.3 \%$ to $24.2 \%$ in viscosity and thermal conductivity for high-temperature ionized air. The conclusion is reached by examining the transport properties using different sets of collision integrals or cross sections in the temperature range from 300 to $30,000 \mathrm{k}[19,29]$.

The initial values and boundary conditions have been well established for the hypersonic interdisciplinary equations $[19,22,23,29]$. The only implicit assumption for the species concentrations on the solid surface is either the non-catalytic or fully catalytic model. However, the mostly widely used condition is simply letting the chemical reaction be controlled by the local thermodynamic condition which is identical to the immediately adjacent domain. In other words, the species concentration on the surface is assumed to be the same as the oncoming stream. Sometimes, such a boundary condition is referred to as super-catalytic; technically, it is assumed that the solid surface is fully catalytic. The other limiting condition is the non-catalytic condition in which the gradient of the species concentration is set to zero.

In describing an ablating surface, the majority of research efforts have been concentrated on the interface boundary conditions either from physical observations or by unique insights. However, by the Reynolds transport theory, the formulation on the interface boundary conditions has been derived from the eigenvector structure of the governing equations [19]. The ejection velocity of the pyrolysis gas and the release vapor rate of the sublimated material on the ablating surface are required as the input. 


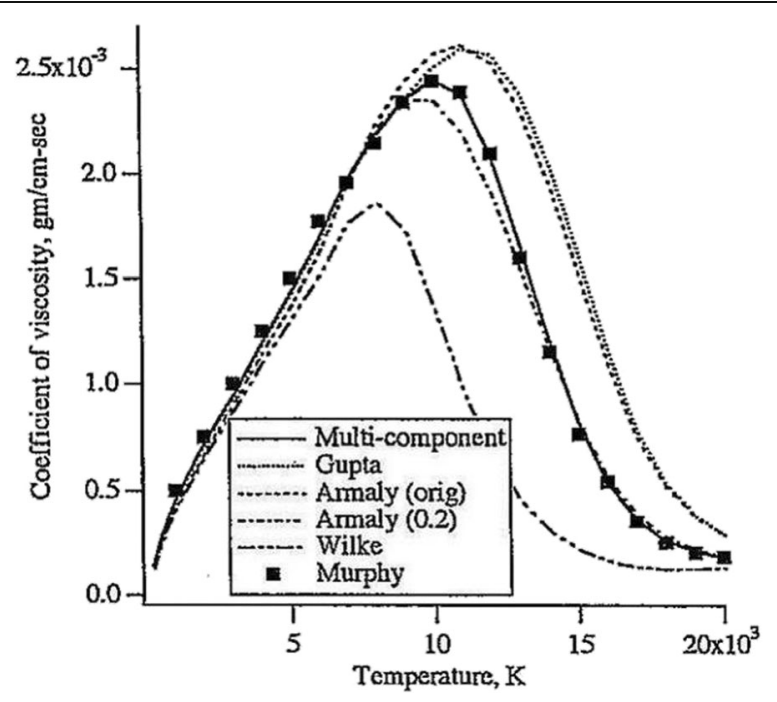

Fig. 16 Viscosity coefficient of ionized air

Therefore the details of gaseous motion through the porous ablating material must be relegated to the research results from a specific ablative material [53, 54].

The interdisciplinary conservation equations, Eqs. (36) through (40), have been routinely applied for simulating the high-enthalpy hypersonic flows. The processes for evaluating the ionized air, nonequilibrium quantum chemical-physics, and radiative energy transfer have been developed by physics-based models. However, the shortcomings of these state-of-the-art models for the nonequilibrium high-temperature chemical kinetic models are known to be woefully limited in their physical fidelity. Nevertheless, the interdisciplinary formulation has been successfully applied to a large group of interplanetary and low-earth reentry simulations, supersonic combustion for Scramjet, and the ion engines performance for engineering purpose [18, 19, 22, 23, 27, 29].

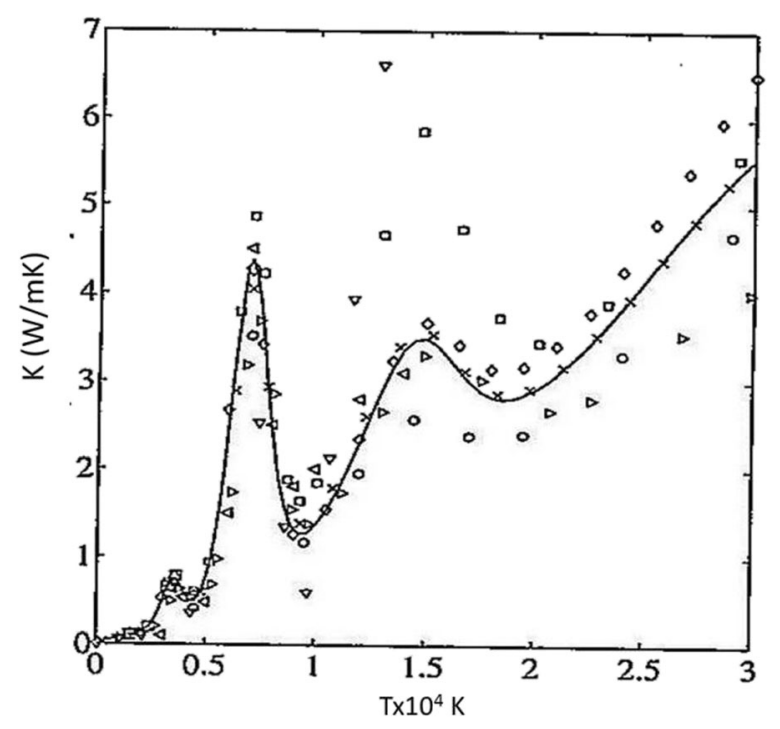

Fig. 17 Thermal conductivity coefficient of equilibrium plasma 
The typical results by the hypersonic interdisciplinary equations with the nonequilibrium quantum chemical-physics models and the transport properties that are derived from the gas kinetic theory are depicted in Figs. 18 and 19. Figure 18 presents the computational results of the entire flow field of the FIRE II space vehicle on a multi-block grid system, solving the radiation rate equation with the spectral line-by-line technique for the emission and absorption coefficients $[29,53]$. These simulations duplicate the reentry trajectory at the time of $1636 \mathrm{~s}$ after an initial recording point. At this instance, the vehicle travels at a speed of $11.31 \mathrm{~km} / \mathrm{s}$, the free stream density and temperature are $\rho_{\infty}=0.857 \times 10^{7} \mathrm{~g} / \mathrm{cm}^{3}$, and $T_{\infty}=210 \mathrm{k}$, and the space vehicle surface temperature is assigned a value of $810 \mathrm{k}$.

On the left-hand-side of the composite figure displays the translational temperature of the air and velocity contours over the space vehicle; the maximum temperature over $25,400 \mathrm{k}$ is confined in the stagnation point region and followed by a rapid expansion over the juncture of the forebody, the afterbody, and continuing into the wake. The vibrational temperatures of nitrogen $\left(\mathrm{T}_{\mathrm{v} 1}\right)$ and oxygen $\left(\mathrm{T}_{\mathrm{v} 2}\right)$ are given by the right-handside of the figure. Both molecular species attain a maximum vibrational temperature of $12,000 \mathrm{k}$ in the thin bow shock layer, but there are distinctive differences between vibrational temperatures of these two species downstream. The vibrational temperature of oxygen molecules decreases continuously toward the wake similar to the translational temperature, but at a 100 times greater value. The vibrational temperature of nitrogen molecules, however, reveals a second peak temperature region in the near wake due to the recompression and nonequilibrium chemical reaction.

The computational simulations of the Stardust sample return capsule generated by the interdisciplinary hypersonic governing equations are exhibited in Fig. 19 for the complete flowfield and the chemical composition in the shock layer. The phenolic impregnated carbon ablator (PIC) has been installed on the front face of the capsule for thermal protection, which increases the complexity for the physical-based computational simulations. Many two and three-dimensional engineering analyses haven been performed for the fastest man-made object in a hypersonic earth reentry [22, 23, 25, 29, 53, 54]. From the trajectory analysis, the reentry vehicle glides at a shallow oscillating angle around eight degrees, which requires the computational simulation conducting in three-dimensional space. The computational results are presented at an altitude of $59.8 \mathrm{~km}$ with the reentry velocity of $11.137 \mathrm{~km} / \mathrm{s}$, and the ambient conditions are characterized by a density of $\rho_{\infty}=2.34 \times$ $10^{-7} \mathrm{~g} / \mathrm{cm}^{3}$ and a temperature of $T_{\infty}=238.5 \mathrm{~K}$.

The velocity topology around the Stardust capsule is described by streamline traces on the right-hand-side of the composite presentation in Fig. 19. The extremely thin shock layer over the forebody of the capsule stands out. The rapid expansions are also noted at the junction of the forebody and afterbody, as well as, in the base region of the capsule. The vortical formation in the near wake region transits from two counterflowing recirculation zones and merges into a biased far-wake structure as the capsule traveling along the trajectory. The basic flow structure follows the rule of the classic aerodynamic theory, however, the air composition in the shock layer and in the near wake is drastically different from the perfect gas model. The air composition in the shock layer indicates the profound and rapid changes by nonequilibrium chemical reactions. In the base region, the air composition by mixing and recombining chemical reactions has affected the local heat transfer drastically. 


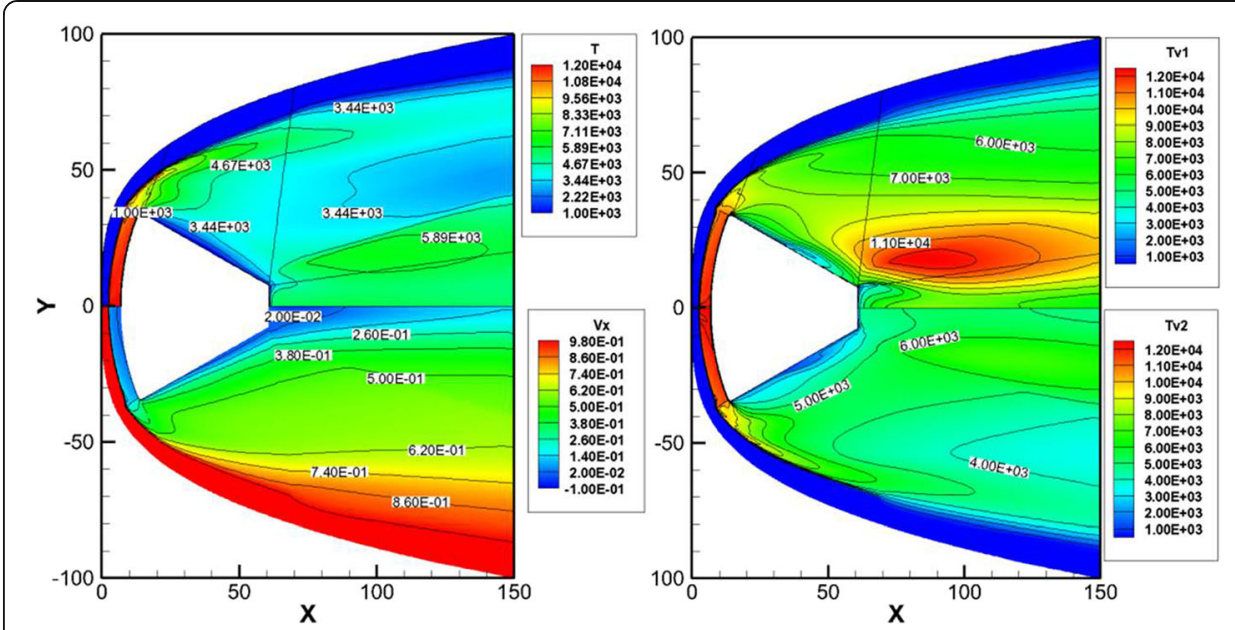

Fig. 18 Translational and vibrational temperature of nitrogen contours over FIRE II space vehicle in Earth reentry, $\mathrm{t}=1636 \mathrm{~s}$

The air composition within the shock layer and along the stagnation streamline is depicted on the right-hand-side of Fig. 19. The maximum translational temperature attains a value as high as $22,750 \mathrm{k}$ and drops to a value of $3250 \mathrm{k}$ in the far wake region. The composition is displayed in the molar fraction of the air mixture, and a total of eighteen species of the ablating gas are presented. The presence of free electrons and a nearly equal amount of positively charged oxygen and nitrogen atoms reveal the air is ionized. The molar fraction of the oxygen molecules is nearly completely depleted in the shock layer and the species of $\mathrm{C}_{3}, \mathrm{HN}$, and $\mathrm{C}^{+}$are presented as a tracing amount of $10^{-4}$ or lower. The rapid recombination and the ablating reactions of different species are noticed in the ablating zone $(x<0.2 \mathrm{~cm})$.

In spite of these engineering accomplishments, only a limited amount of results from the hypersonic interdisciplinary governing equations have been verified by comparing with the flight and the ground-based experimental data. For the latter, an exemplified collection of the high-enthalpy hypersonic flows experimental facilities and their measurement techniques can be found in reference [55-57]. Nevertheless, the simulated
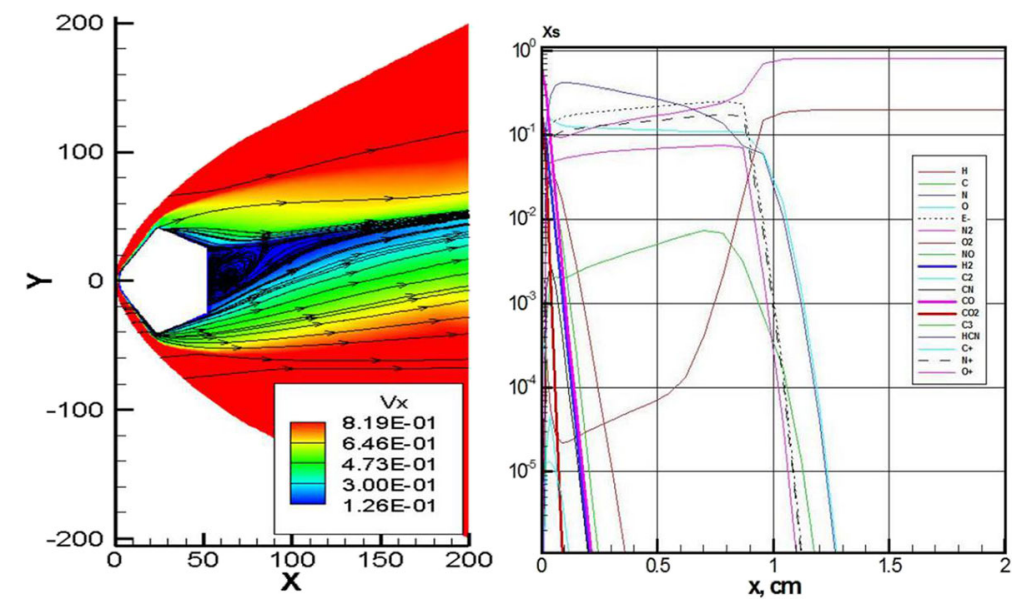

Fig. 19 Computational results of Stardust sample return capsule 
computational results still can duplicate the essential physics to be retained for the engineering purpose. A substantial amount of basic research efforts are urgently and critically needed to build on the basic knowledge of a lasting scientific value for the high enthalpy hypersonic flows.

Some promising basic research activities have been initiated to address the pacing item in hypersonic flow research by combining the theoretical knowledge with the support from the high performance computational technology $[18,19]$. The ab initio or the first principle approach offers the opportunity for a scientific breakthrough, which is based on the Born-Oppenheimer approximation by separating the wave function of the nuclei from the electron [58]. These solutions are focused on the energy distribution by quantum mechanics. The Schrödinger equation is first solved for a single electron then adds the internucleus repulsive electronic energy to get the total internal energy of a molecule. In order to examine these phenomena, the entire potential energy surfaces (PES) must be constructed for chemical-physics interactions [58, 59]. In fact, the PES is a plot of the collective nuclei and electronic energy versus the molecule geometric coordinates. In other words, the PES provides a visualizing and understanding of the relationship between potential energy and molecular geometry. Therefore, the $a b$ initio approach actually determines the structure, the energy of molecules, and the transition states involving chemical reactions. Impressive accomplishments have been achieved for constructing the critical potential surface, which enables the prediction of the critical point for transition [18, 58-60].

An example of the potential energy surface is displayed by Fig. 20. The contours of the PES are constructed from 3326 ab initio solutions by solving the Schrödinger equation for a N-N bond nitrogen molecule that shows the double barriers of two transition states connected by a shallow energy well [59]. The two barriers are symmetric with respect to the interchange of two nitrogen atoms, and the bond distance is less than 3 Angstrom, and the calculated bond angle is fixed at $119^{\circ}$. The inset of this figure illustrates the partition of atoms $\mathrm{A}$ and $\mathrm{B}$ either in a heteronuclear or a homonuclear molecule [58]. These atoms are separated by a zero-flux surface $S$ that passes through the critical saddle point on the bond path C. According to the critical point theory by Eyring, it's the location for transition to occur [60]. This type of incisive knowledge is absolutely crucial for advancing our knowledge by basic research for high-enthalpy hypersonic flows.

\section{Concluding remarks}

Hypersonic flows always exist in a high-enthalpy environment by strong shock wave compression. Under this condition, the flow medium undergoes significant change, which is no longer accurately describable by the perfect gas assumption. Although the basic aerodynamic behavior remains mostly unaltered, the critical thermodynamic properties however require a multidisciplinary description. When the air is ionized, the propagation of electromagnetic waves is presented and introduces added external forces and energy transfer mechanisms. Thus, hypersonic flows are governed by aerodynamics, electromagnetics, nonequilibrium quantum chemical-physics, and nonequilibrium radiation. The current interdisciplinary formulation is limited to the engineering applications for supersonic combustion, electric propulsion, and space vehicle earth reentry. 


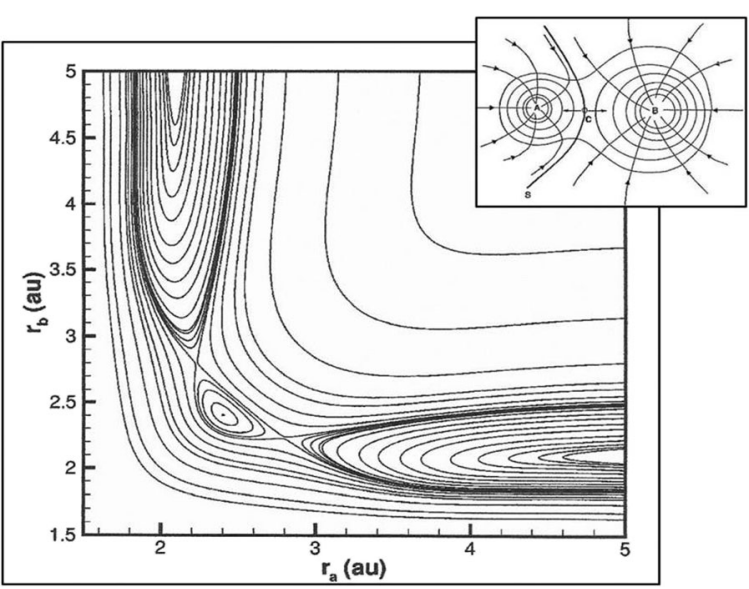

Fig. 20 Potential energy surface of $\mathrm{N}_{2}+\mathrm{N}$ exchange reaction

This approach falls short to reach scientific accuracy for the last few frontier of aerodynamics and for future innovations.

The challenge of these pacing items for the high-enthalpy hypersonic flows resides in the delineation of flow medium at a nonequilibrium high energy state. It is necessary to overcome our knowledge shortcomings for describing the nonequilibrium processes in chemical kinetics, quantum transitions, and optical properties of the highly excited air. In short term, the development of a specific data base is critical for the improved accuracy of chemical reacting rate constants, the intermolecular potential energy for transport properties, and the emission and absorption coefficients for radiation. Ultimately, the fundamental knowledge must be built on basic research by implementing the inelastic collision model including quantum chemical-physics to the gas kinetic theory, and by adopting the abi initio approach to gain insights for the transient phenomena; these basic research efforts are arduous and formidable but only of a lasting value.

\section{Acknowledgements}

In preparation of the manuscript, authors have received constant encouragement and inspiration for pursuing of excellence from Prof. Chuanjun Yan (deceased) of the Northwestern Polytechnical University of China. This manuscript is humbly dedicated to his memory.

\section{Authors' contributions}

JS conceived of the study, and drafted the manuscript. JS performed the review of the classic hypersonic theories, modelled the electron impact ionization, and conducted the computational simulations and verifications for the ionized air around the reentry RAM-C-II probe, nonequilibrium radiative heat transfer to FIRE II probe, as well as, the analysis for the Stardust sample return capsule by the hypersonic interdisciplinary governing equations. HY carried out the nonequilibrium chemical kinetics computations, and participated in the sequence alignment. The authors read and approved the final manuscript. The manuscript is a piece of original work by the authors, and is prepared exclusively for the journal Advances in Aerodynamics.

\section{Funding}

N/A

\section{Availability of data and materials}

All the data and materials of our manuscript are either generated by the authors or are available from the open literacy with appropriated citations.

\section{Competing interests}

The manuscript does not represent any competing interests.

\section{Author details}

${ }^{1}$ Wright State University, 3640 Colonel Glenn Highway, Dayton, OH 45435, USA. ${ }^{2}$ School of Power and Energy,

Northwestern Polytechnical University, 127 West Youyi Road, Xi'an 710072, China. 
Received: 4 May 2020 Accepted: 19 June 2020

Published online: 07 August 2020

\section{References}

1. Hayes WD, Probstein RF (1959) Hypersonic flow theory. Academic Press, New York

2. Clarke JF, McChesney M (1964) The dynamics of real gases. Butterworth Inc., Washington D.C

3. Tsien HS (1946) Similarity laws of hypersonic flows. J Math Phys 25:247-251

4. Il'yushin AA (1956) The law of plane sections in the aerodynamics of high supersonic speeds. PMM 20:733-755

5. Newton I (1934) The mathematical principal of natural philosophy. trans: Motte A, (1729), revised: Cajori A. University of California Press, Berkeley

6. Lees L (1955) Hypersonic flow, 5th international aeronautical conference. AlAA, Los Angeles, pp 241-276

7. Chernyi GG (1961) Introduction to hypersonic flow. Academic Press, New York

8. Sychev W (1960) Three-dimensional hypersonic gas flow past slender bodies at high angles of attack. PMM 24(2):205-212

9. Sedov LI (1945) On certain unsteady motions of a compressible fluid. PMM 9:293-311

10. Taylor GI (1950) The formation of a blast wave by a very intense explosion I. Theoretical discussion. Proc R Soc Lond. A 201:159-174

11. Lukasiewicz J (1962) Blast-hypersonic flow analogy theory and application. Am Rocket Soc J 32(9):1341-1346

12. Bertram MH (1956) Tip-bluntness effects on cone pressures at M=6.85. J Aero Sci 23:898-900

13. Dorodnitsyn AA (1942) Laminar boundary layer in compressible fluid. Dokl Akad Nauk SSSR 34:214-219

14. Mangler W (1945) Boundary layers on bodies of revolution in symmetrical flow, (Ber. Aerodyn. Versuchsanst. Goett., Rept. 45/A/17, 1945). J. of Applied Mat and Mech, 28(4):97-103

15. Van Driest ER (1956) The problem of aerodynamic heating. Aeronautical Eng Review, pp 26-41

16. Kemp NH, Rose PH, Detra RW (1959) Laminar heat transfer around blunt bodies in dissociated air. J Aerospace Sci 26(7): $421-430$

17. Shang JS (2010) Fundamental of hypersonic flows. In: Encyclopedia of aerospace engineering. Wiley, New York, pp 36-1-12 ISBN: 9780470754405

18. Shang JS (2019) Landmarks and new frontiers of computational fluid dynamics. Adv. Aerodyn. 1:5 https://doi.org/10. 1186/s42774-019-0003-x

19. Shang JS (2016) Computational electromagnetics-aerodynamics. Wiley, Hoboken

20. Mitchner M, Kruger CH (1973) Partially ionized gases. Wiley, New York

21. Dunn MG, Kang SW (1973) Theoretical and experimental studies of reentry plasmas. NASA CR 2232

22. Olynick D, Chen Y, Tauber M (1999) Aerothermodynamics of the Stardust sample return capsule. J Spacecraft Rockets 36(3):442-462

23. Park C (1993) Review of chemical-kinetic problems of future NASA missions, I. Earth entries. J Thermophys Heat Transfer 7(3): 385-398

24. Shang JS, Huang PG, Yan H, Surzhikov ST (2009) Computational electrodynamic simulation of direct current discharge. J Appl Phys 105(2):023303

25. Shang JS, Andrienko DA, Huang PG, Surzhikov ST (2014) A computational approach for hypersonic nonequilibrium radiation utilizing space partition algorithm and Gauss quadrature. J Comp Physics 266:1-21

26. Raizer YP (1991) Gas discharge physics. Springer-Verlag, Berlin

27. Jahn RG (1968) Physics of electric propulsion. McGraw-Hill, New York

28. Herzberg G (1944) Atomic spectra and atomic structure, $2^{\text {nd }}$ edn. Dover Publications, New York

29. Shang JS, Surzhikov ST (2018) Plasma dynamics for aerospace engineering. Cambridge University Press, Cambridge

30. Zel'dovich Ya B, Raizer YP (2002) Physics of shock waves and high-temperature hydrodynamic phenomena. Dover publications, Mineola

31. Langmuir I (1928) Oscillations in ionized gases. Proc Natl Acad Sci 14(8):627-637

32. Bittencourt JA (1986) Fundamentals of plasma physics. Pergamon Press, Oxford

33. Alfven H (1950) Cosmical electrodynamics. Clarendon Press, Oxford

34. Kraus JD (1953) Electromagnetics, 1st edn. McGraw-Hill, New York

35. Sutton GW, Sherman A (1965) Engineering magnetohydrodynamics. McGraw-Hill, New York

36. Hall EH (1879) On a new action of the magnet on electric currents. Am J Math 2(3):287-292

37. Mahalingam S (2007) Particle based plasma simulation for an ion engine discharge. Dissertation, Wright State University

38. Ziemer RW (1959) Experimental investigation in magneto-aerodynamics. J Am Rocket Soc 29(9):642-647

39. Kong JA (1986) Electromagnetic wave theory. Wiley, New York

40. Modest MF (1993) Radiative heat transfer. McGraw-Hill, Inc., New York

41. Siegel R, Howell JR (2002) Thermal radiation heat transfer, 4th edn. Taylor \& Francis, New York

42. Surzhikov ST (2002) Radiation modeling and spectral data. Von Karman lecture series. 2002-2007. von Karman Institute for Fluid Dynamics, Rhode-ST-Genese Belgium

43. Sakami M, Charette A, Le Dez V (1998) Analysis of radiative heat transfer in enclosures of complex geometry using the discrete ordinates method. Proceedings of the 2nd international symposium on radiation transfer, Kusadasi, pp 253-270

44. Kong R, Ambrose M, Spanier J (2008) Efficient, automated Monte Carlo methods for radiation transport. J Comput Phys 227(22):9463-9476

45. Mazouffre S, Caubet-Hilloutou V, Dudeck M, Pawelec E (2003) Operating characteristics of the SR5 hypersonic plasma wind-tunnel. International workshop on radiation of high temperature gases in atmospheric entry, Lisbon

46. Landau L, Teller E (1936) Zur theorie der schalldispersion. Physik Z Sowjetunion 10(1):34-43

47. Millikan RC, White DR (1963) Systematics of vibrational relaxation. J Chem Phys 39(12):3209-3213

48. Chernyi GG, Losev SA, Macheret SO, Potapkin BV (2004) Physical and chemical processes in gas dynamics. In: Zarchan P (ed) Physical and chemical kinetics and thermodynamics. American Institute of Aeronautics and Astronautics, Reston

49. Hirschfelder JO, Curtiss CF, Bird RB (1954) Molecular theory of gases and liquids, $2^{\text {nd }}$ edn. Wiley, New York

50. Capitelli M, Gorse C, Longo SJ, Giordano D (2000) Collision integrals of high-temperature air species. J Thermophys Heat Transfer 14(2):259-268 
51. Palmer G, Wright M (2003) Comparison of methods to compute high temperature viscosity. J Thermophys Heat Transfer 17(2):232-239

52. D'Angola A, Colonna G, Gorse C, Capitelli M (2008) Thermodynamic and transport properties in equilibrium air plasma in a wide pressure and temperature range. Eur Phys J D 46:129-150

53. Shang JS, Surzhikov ST (2012) Nonequilibrium radiative hypersonic flow simulation. Prog Aerosp Sci 53:46-65

54. Chen YK, Milos FS (2005) Navier-Stokes solutions with finite rate ablation for planetary mission earth reentries. J Spacecraft Rockets 42(6):961-970

55. Lu F, Marren D (2002) Advanced hypersonic test facilities, Progress in astronautics and aeronautics. Vol 198. AIAA, Reston

56. Simmons JM (1995) Measurement techniques in high-enthalpy hypersonic facilities. Exp Therm Fluid Sci 10(4):454-469

57. Seleznev RK, Surzhikov ST, Shang JS (2019) A review of the scramjet experimental data base. Prog Aerosp Sci 106:43-70

58. Lewars EG (2011) Computational chemistry: Introduction to the theory and applications of molecule and quantum mechanics. Springer, New York

59. Wang D, Stallcop J, Huo W, Dateo C, Schwenke D, Partridge H (2003) Quantal study of the exchange reaction for $\mathrm{N}+\mathrm{N}_{2}$ using an ab initio potential energy surface. J Chem Phys 118(5):2186-2189

60. Eyring H, Walter J, Kimball GE (1944) Quantum chemistry. Wiley, New York

\section{Publisher's Note}

Springer Nature remains neutral with regard to jurisdictional claims in published maps and institutional affiliations.

Submit your manuscript to a SpringerOpen ${ }^{\circ}$ journal and benefit from:

- Convenient online submission

- Rigorous peer review

- Open access: articles freely available online

- High visibility within the field

- Retaining the copyright to your article

Submit your next manuscript at $\boldsymbol{\nabla}$ springeropen.com 